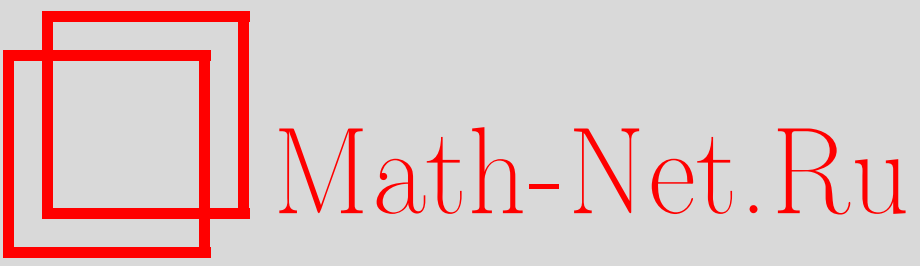

А. Г. Сергеев, Гармонические отображения в однородные римановы многообразия: твисторный подход, УМН, 2004, том 59, выпуск 6, 177-200

DOI: https://doi.org/10.4213/rm801

Использование Общероссийского математического портала Math-Net.Ru подразумевает, что вы прочитали и согласны с пользовательским соглашением

http://www.mathnet.ru/rus/agreement

Параметры загрузки:

IP : 18.234 .197 .8

26 апреля 2023 г., 17:06:04 


\section{ГАРМОНИЧЕСКИЕ ОТОБРАЖЕНИЯ В ОДНОРОДНЫЕ РИМАНОВЫ МНОГООБРАЗИЯ: ТВИСТОРНЫЙ ПОДХОД}

\section{А. Г. СЕРГЕЕВ}

Статья посвящена твисторному подходу к исследованию гармонических отображений $\varphi: M \rightarrow N$ из римановых поверхностей $M$ в римановы многообразия $N$. Идея подхода заключается в том, чтобы для заданного риманова многообразия $N$ построить так назьваемое твисторное расслоение $\pi: Z \rightarrow N$, где $Z$ - почти комплексное многообразие, которое обладает следующим свойством: проекция $\pi \circ \psi: M \rightarrow N$ любого почти голоморфного отображения $\psi: M \rightarrow Z$ является гармоническим отображением. Для широких классов римановых многообразий $N$ твисторный подход позволяет построить указанным образом все гармонические отображения $\varphi: M \rightarrow N$ и тем самым свести исходную вещественную задачу описания гармонических отображений в римановы многообразия к комплексной задаче описания почти голоморфиых отображений в почти комплексные многообразия. В статье подробно рассмотрены следующие классы однородных римановых многообразий $N$, к которым применим твисторньй метод, - это компактные группы Ли, пространства петель таких групп и грассмановы многообразия, включая гильбертов грассманиан.

Библиография: 11 названий.

\section{СОДЕРЖАНИЕ}

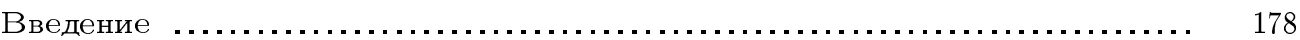

$\S 1$. Гармонические отображения и интегрируемые системы $\ldots . . \ldots \ldots . .179$

1.1. Гармонические отображения римановых многообразий ............. 179

1.2. Гармонические отображения в компактные группы Ли (конструкция Уленбек) .............................................. 180

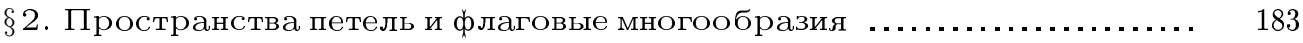

2.1. Пространство петель компактной группы Ли . . . . . . . . . . . . . . . 183

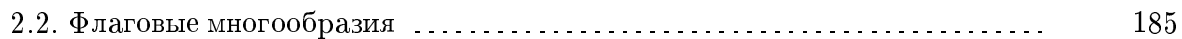

$\S 3$. Твисторные конструкции гармонических отображений $\ldots . . . . . . . . .189$

3.1. Гармонические отображения в грассмановы многообразия и симметрические пространства . ..................................... 189

3.2. Пространство петель как твисторное расслоение . . . . . . . . . . . . . 193

3.3. Гармонические отображения в гильбертов грассманиан и пространства

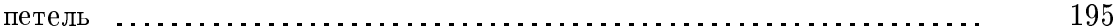

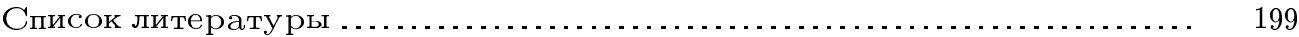




\section{Введение}

Статья посвяшена исследованию гармонических отображений $\varphi: M \rightarrow N$ из римановых поверхностей $M$ в римановы многообразия $N$ с помошњю твисторного подхода. Идея этого подхода состоит в том, чтобы свести вещественную задачу описания гармонических отображений к комплексной задаче описания голоморфных отображений в почти комплексные многообразия. А именно, для заданного риманова многообразия строится твисторное расслоение $\pi: Z \rightarrow N$, где $Z$ - почти комплексное многообразие, которое обладает следуюшим свойством: для любого почти голоморфного отображения $\psi: M \rightarrow Z$ его проекция $\varphi:=\pi \circ \psi: M \rightarrow N$ является гармоническим отображением. Общая теория твисторных пространств представлена в [6], в данной статье мы ограничиваемся подробньм изложением твисторного подхода для важных классов однородных многообразий $N$, а именно компактных групп Ли, их пространств петель и грассмановых многообразий, включая гильбертов грассманиан.

Твисторньй подход к исследованию гармонических отображений римановых поверхностей в компактные группы Ли $G$ (на примере унитарной группы) был предложен Уленбек в работе [10] (хотя твисторная терминология и не использовалась в этой статье). Мы излагаем конструкцию Уленбек в п. 1.2. В ее основе лежит представление гармонического уравнения в виде уравнения нулевой кривизны для семейства связностей, зависящих от спектрального параметра $\lambda$. На языке твисторов это означает, что естественное отображение $\Omega G \rightarrow G$, сопоставляюшее петле $\gamma \in \Omega G$ ее значение в точке $-1 \in S^{1}$, является твисторным расслоением над группой $G$ (как это объясняется в п. 3.2). Более того, в рассматриваемом случае любое гармоническое отображение $\varphi: M \rightarrow G$ может быть реализовано как проекция почти голоморфного отображения $\psi: M \rightarrow \Omega G$. С помошю подхода Уленбек можно также построить преобразование типа Бэклунда на пространстве гармонических отображений, которое позволяет "размножать" гармонические отображения.

В следуюшем параграфе приводятся основные необходимые для дальнейшего сведения о пространствах петель компактных групп Ли и флаговых многообразиях (более подробно об этом см. в [9]).

Третий параграфф посвящен твисторным конструкциям гармонических отображений. В п. 3.1 строится целая серия твисторных расслоений над грассмановыми многообразиями, ассоциированных с флаговыми многообразиями. В случае римановой сферы $M=\mathbb{C P}^{1}$ твисторный подход позволяет получить любые гармонические отображения из $M$ в грассмановы многообразия. Указанная твисторная конструкщия переносится на так назьваемые внутренние симметрические пространства, возникающие как базы однородных расслоений флаговых многообразий. В п. 3.2 дается твисторная интерпретация конструкции Уленбек из п. 1.2. В п. 3.3 рассматриваются гармонические отображения в грассманиан гильбертова пространства и строятся аналоги флаговых расслоений над ними (предложение 3.9 ). Так как пространство петель $\Omega G$ допускает изометрическое вложение в гильбертов грассманиан, этот класс содержит, в частности, все гармонические отображения в $\Omega G$. Последние представляют особьй интерес ввиду их связи с уравнениями Янга-Миллса на $\mathbb{R}^{4}$.

Работа выполнена при частичной финансовой поддержке Российского фонда фундаментальных исследований (гранты № 04-01-00236, 02-02-04002), программы поддержки ведущих научных школ (грант НШ-1542.2003.1) и программы фундаментальных исследований Президиума РАН "Математические методы в нелинейной динамике". 


\section{$\S$ 1. Гармонические отображения и интегрируемые системы}

1.1. Гармонические отображения римановых многообразий. Пусть $\varphi: M \rightarrow N$ есть гладкое отображение римановых многообразий. Его дифференщиал $\varphi_{*}: T M \rightarrow T N$ можно рассматривать как сечение $d \varphi$ расслоения $T^{*} M \otimes \varphi^{-1}(T N)$, где $\varphi^{-1}(T N)$ - обратный образ касательного расслоения $T N$ при отображении $\varphi$ : слой $\varphi^{-1}(T N)_{p}$ в точке $p \in M$ есть $T_{\varphi(p)} N$. Наделим расслоение $T^{*} M \otimes \varphi^{-1}(T N)$ римановой метрикой, индуцированной римановыми метриками на $M$ и $N$, и обозначим через $|d \varphi|$ норму $d \varphi$, вычисленную в указанной метрике. В локальных координатах $\left(x^{i}\right)$ в точке $p \in M$ и $\left(u^{\alpha}\right)$ в точке $\varphi(p) \in N$ эта норма равна

$$
|d \varphi(p)|^{2}=\sum_{i, j} \sum_{\alpha, \beta} g^{i j} \frac{\partial \varphi^{\alpha}}{\partial x^{i}} \frac{\partial \varphi^{\beta}}{\partial x^{j}} h_{\alpha \beta},
$$

где $\varphi^{\alpha}=\varphi^{\alpha}(x)$ - компоненты отображения $\varphi,\left(g_{i j}\right)$ и $\left(h_{\alpha \beta}\right)$ - метрические тензоры многообразий $M$ и $N$ соответственно, а $\left(g^{i j}\right)$ - матрица, обратная к $\left(g_{i j}\right)$.

Определим әнергию отображения $\varphi$ по формуле

$$
E(\varphi)=\frac{1}{2} \int_{M}|d \varphi(p)|^{2} \operatorname{vol}_{g},
$$

где $\operatorname{vol}_{g}$ - элемент объема в метрике $g$ на $M$. Отображение $\varphi$ назьвается гармоническим, если оно является экстремальным для функционала $E(\varphi)$ по отношению к гладким вариациям $\varphi$ с компактным носителем.

Уравнение Эйлера-Лагранжа для $E(\varphi)$ имеет вид

$$
\operatorname{tr}(\nabla d \varphi)=0
$$

где оператор $\tau_{\varphi}:=\operatorname{tr}(\nabla d \varphi)$ назьвается полем напряжений отображения $\varphi$. В локальном ортонормированном базисе $\left\{X_{j}\right\}$ касательного расслоения $T M$ в окрестности точки $p \in M$ он задается формулой

$$
\operatorname{tr}(\nabla d \varphi)_{p}=\sum_{j}\left({ }^{M} \nabla_{X_{j}} d \varphi\right)\left(X_{j}\right)
$$

где ${ }^{M} \nabla$ - связность Леви-Чивита многообразия $M$. Пользуясь этим выражением, вьпишем уравнения (1.2) в локальных координатах $\left(x^{i}\right)$ на $M$ и $\left(u^{\alpha}\right)$ на $N$ :

$$
\begin{gathered}
\sum_{i, j} g^{i j}\left\{\frac{\partial^{2} \varphi_{\gamma}}{\partial x_{i} \partial x_{j}}-\sum_{k}{ }^{M} \Gamma_{i j}^{k} \frac{\partial \varphi_{\gamma}}{\partial x_{k}}+\sum_{\alpha, \beta}{ }^{N} \Gamma_{\alpha \beta}^{\gamma}(\varphi) \frac{\partial \varphi_{\alpha}}{\partial x_{i}} \frac{\partial \varphi_{\beta}}{\partial x_{j}}\right\} \\
=\Delta_{M} \varphi_{\gamma}+\sum_{i, j} g^{i j} \sum_{\alpha, \beta}{ }^{N} \Gamma_{\alpha \beta}^{\gamma}(\varphi) \frac{\partial \varphi_{\alpha}}{\partial x_{i}} \frac{\partial \varphi_{\beta}}{\partial x_{j}}=0,
\end{gathered}
$$

где ${ }^{M} \Gamma_{i j}^{k},{ }^{N} \Gamma_{\alpha \beta}^{\gamma}$ - символы Кристоффеля связностей Леви-Чивита ${ }^{M} \nabla$ на $M$ и ${ }^{N} \nabla$ на $N$ соответственно, а $\Delta_{M}$ - оператор Лапласа-Бельтрами многообразия $M$. В случае $N=\mathbb{R}^{n}$ уравнение (1.3) становится линейным и преврашается в обычное уравнение Лапласа-Бельтрами на многообразии $M$. 
Нас будет особо интересовать случай, когда $M$ есть риманова поверхность, а $N$ кэлерово многообразие. В этой ситуации мы будем предполагать, что многообразия $M$ и $N$ наделены комплексными структурами, совместимыми с римановыми метриками на данных многообразиях. Пусть $\varphi: M \rightarrow N$ есть гладкое отображение римановой поверхности $M$ в кэлерово многообразие $N$. Продолжим дифференциал $d \varphi$ комплексно-линейно на комплексифицированное расслоение $T^{\mathbb{C}} M \otimes \varphi^{-1}\left(T^{\mathbb{C}} N\right)$ и представим его в виде

$$
d \varphi=\delta \varphi+\bar{\delta} \varphi
$$

где $\delta \varphi$ есть сечение расслоения $T^{1,0} M \otimes \varphi^{-1}\left(T^{\mathbb{C}} N\right)$, а $\bar{\delta} \varphi-$ сечение $T^{0,1} M \otimes \varphi^{-1}\left(T^{\mathbb{C}} N\right)$. Тогда условие гармоничности отображения $\varphi$ примет вид

$$
\bar{\delta} \delta \varphi=\nabla_{\partial / \partial \bar{z}} d \varphi\left(\frac{\partial}{\partial z}\right)=0 \Longleftrightarrow \delta \bar{\delta} \varphi=\nabla_{\partial / \partial z} d \varphi\left(\frac{\partial}{\partial \bar{z}}\right)=0
$$

где через $\nabla$ обозначена связность на расслоении $\varphi^{-1}\left(T^{\mathbb{C}} N\right)$, индушированная связностью Леви-Чивита ${ }^{N} \nabla$ на $N$.

Для кэлерова многообразия $N$ дифференциал $d \varphi$ можно разложить и далее, пользуясь представлением

$$
\delta \varphi=\partial^{\prime} \varphi+\overline{\partial^{\prime \prime} \varphi}, \quad \bar{\delta} \varphi=\partial^{\prime \prime} \varphi+\overline{\partial^{\prime} \varphi}
$$

где $\partial^{\prime} \varphi$ - сечение $T^{1,0} M \otimes \varphi^{-1}\left(T^{1,0} N\right)$, а $\partial^{\prime \prime} \varphi$ - сечение $T^{0,1} M \otimes \varphi^{-1}\left(T^{1,0} N\right)$. Условие гармоничности (1.4) в этих обозначениях принимает вид

$$
\bar{\delta} \partial^{\prime} \varphi=0 \Longleftrightarrow \delta \partial^{\prime \prime} \varphi=0
$$

Напомним теорему Кошуля-Мальгранжа (см. [7]), согласно которой для любого комплексного векторного расслоения $E$ над римановой поверхностью $M$, наделенного связностью $\nabla$, существует единственная комплексная структура $J$ на $E$, относительно которой $E \rightarrow M$ является голоморфным векторныл расслоением, а $\bar{\partial}_{J}$-оператор совпадает с $(0,1)$-составляющей $\nabla^{0,1}$ связности $\nabla$. Указанную комплексную структуру $J$ будем называть $K M$-структурой.

В ее терминах первое из условий гармоничности (1.4) означает, что $\delta \varphi$ является голоморфным сечением расслоения $\varphi^{-1}\left(T^{\mathbb{C}} N\right)$ относительно KM-структурын на $\varphi^{-1}\left(T^{\mathbb{C}} N\right)$, индуиированной связностью ${ }^{N} \nabla$. Аналогично, первое из условий $(1.5)$ означает, что $\partial^{\prime} \varphi-$ голоморфное сечение расслоения $\varphi^{-1}\left(T^{1,0} N\right)$.

1.2. Гармонические отображения в компактные группы Ли (конструкция Уленбек). Пусть $G$ есть компактная группа Ли, снабженная двусторонне инвариантной (относительно присоединенного представления) римановой метрикой. Обозначим через $\mu$ форму Картана-Маурера на $G$, т.е. тавтологическую 1-форму на $G$ со значениями в алгебре Ли g группы $G$. Она задается формулой: $\mu(\xi)=\xi$ для любого элемента $\xi \in \mathfrak{g}$, рассматриваемого как левоинвариантное векторное поле на $G$. Тогда

$$
d \mu+\frac{1}{2} \mu \wedge \mu=0,
$$

где 2-форма $\mu \wedge \mu$ на $G$ определяется посредством

$$
\frac{1}{2}(\mu \wedge \mu)(\xi, \eta):=[\mu(\xi), \mu(\eta)] \text { для } \xi, \eta \in \mathfrak{g} .
$$


Тем самьм $\mu$ является формой плоской связности на $G$.

Пусть теперь $\varphi: M \rightarrow G$ есть гладкое отображение римановой поверхности $M$ в групп $G$. Обозначим через $\alpha:=\varphi^{*} \mu$ обратный образ формы Картана-Маурера при отображении $\varphi$. Тогда $\alpha$ есть $\mathfrak{g}$-значная 1-форма на $M$, удовлетворяюшая равенству

$$
d \alpha+\frac{1}{2} \alpha \wedge \alpha=0
$$

Иначе говоря, форма $\alpha$ задает плоскую связность на расслоении $\varphi^{-1}(T G)$. Продолжая форму $\alpha$ на комплексифицированное расслоение $\varphi^{-1}\left(T^{\mathbb{C}} G\right)$, мы можем записать ее в локальной карте на $M$ с локальной координатой $z$ в виде $\alpha=A_{z} d z+A_{\bar{z}} d \bar{z}$. Условие гармоничности отображения $\varphi$ переписьвается в терминах $\alpha$ в виде

$$
\bar{\partial} A_{z}+\partial A_{\bar{z}}=0
$$

а условие (1.6) дает соотношение

$$
\bar{\partial} A_{z}-\partial A_{\bar{z}}+2\left[A_{\bar{z}}, A_{z}\right]=0 .
$$

Соотношения (1.7) можно записать в эквивалентном, но более симметричном виде

$$
\bar{\partial} A_{z}+\left[A_{\bar{z}}, A_{z}\right]=0, \quad \partial A_{\bar{z}}-\left[A_{\bar{z}}, A_{z}\right]=0 .
$$

Сопоставим форме $\alpha$ семейство форм $\alpha_{\lambda}$, зависящее от параметра $\lambda \in \mathbb{C}^{*}:=\mathbb{C} \backslash\{0\}$ :

$$
\alpha_{\lambda}=\frac{1}{2}\left(1-\lambda^{-1}\right) A_{z} d z+\frac{1}{2}(1-\lambda) A_{\bar{z}} d \bar{z}
$$

Заметим, что семейство $\alpha_{\lambda}$ включает в себя исходную форму $\alpha$ при $\lambda=-1$.

Лемма 1.1 (Уленбек [10]). Отображение $\varphi: M \rightarrow G$ гармонично тогда и только тогда, когда связность на расслоении $\varphi^{-1}(T G)$, задаваемая формой $\alpha_{\lambda}$, яөляется плоской при всех $\lambda \in \mathbb{C}^{*}$. Иначе говоря,

$$
d \alpha_{\lambda}+\frac{1}{2} \alpha_{\lambda} \wedge \alpha_{\lambda}=0 \quad \text { для любого } \lambda \in \mathbb{C}^{*} .
$$

ДокАЗАТЕЛЬСТВО. В терминах локальной комплексной координаты $z$ на $M$ имеем

$$
\begin{aligned}
& 2\left(d \alpha_{\lambda}+\frac{1}{2} \alpha_{\lambda} \wedge \alpha_{\lambda}\right) \\
& \quad=\left(1-\lambda^{-1}\right) \bar{\partial} A_{z} \wedge d z+(1-\lambda) \partial A_{\bar{z}} \wedge d \bar{z}+\left[\left(1-\lambda^{-1}\right) A_{z},(1-\lambda) A_{\bar{z}}\right] \\
& \quad=\lambda\left(-\partial A_{\bar{z}}+\left[A_{\bar{z}}, A_{z}\right]\right)+\left(-\bar{\partial} A_{z}+\partial A_{\bar{z}}-2\left[A_{\bar{z}}, A_{z}\right]\right)+\frac{1}{\lambda}\left(\bar{\partial} A_{z}+\left[A_{\bar{z}}, A_{z}\right]\right) .
\end{aligned}
$$

Коэффициенты при $\lambda$ и $\frac{1}{\lambda}$ равны нулю ввиду соотношений (1.8), а постоянньй по $\lambda$ член равен нулю ввиду $(1.7 \mathrm{~b})$. Лемма доказана.

С формой $\alpha_{\lambda}$ связана система линейных уравнений типа Захарова-Шабата, имеюшая вид

$$
d E_{\lambda}=E_{\lambda} \alpha_{\lambda} \Longleftrightarrow E_{\lambda}^{-1} d E_{\lambda}=\alpha_{\lambda} \text { при } \lambda \in \mathbb{C}^{*},
$$

которую в локальной карте можно переписать в виде

$$
\bar{\partial} E_{\lambda}=(1-\lambda) E_{\lambda} A_{\bar{z}}, \quad \partial E_{\lambda}=\left(1-\lambda^{-1}\right) E_{\lambda} A_{z}, \quad \lambda \in \mathbb{C}^{*} .
$$

Уравнение нулевой кривизны (1.9) является условием совместности системы (1.10) (или (1.11)). Поэтому, фиксируя произвольную отмеченную точку $p_{0} \in M$, мы можем вывести отсюда утверждение о разрешимости системы (1.10). 
ПРЕДЛОЖЕНИЕ 1.2 (УЛенбек [10]). Пусть $\varphi: M \rightarrow G-$ иентрированное (т.е. $\left.\varphi\left(p_{0}\right)=e \in G\right)$ гармоническое отображсение односвязной римановой поверхности $M$ в компактную группу Ли $G$. Тогда существует единственное решение $E: M \times \mathbb{C}^{*} \rightarrow G^{\mathbb{C}}$ системы (1.10), удовлетворяющее условиям:

$$
E_{1}=e, \quad E_{-1}=\varphi, \quad E_{\lambda}\left(p_{0}\right)=e \quad \partial \Omega_{я} \quad \text { всех } \quad \lambda \in \mathbb{C}^{*}
$$

Более того, $E_{\lambda}$ голоморфно зависит от $\lambda \in \mathbb{C}^{*} u E_{\lambda} \in G$ при $\lambda \in S^{1}$.

Заметим, что семейство $E_{\lambda}$ при каждом $\lambda \in \mathbb{C}^{*}$ задает тривиализацию расслоения $\varphi^{-1}\left(T^{\mathbb{C}} G\right)$, рассматриваемого как голоморфное расслоение относительно канонической комплексной КМ-структуры, порождаемой формой Картана-Маурера $\alpha$.

Обратно, справедливо следующее предложение.

ПРЕДЛОЖЕНИЕ 1.3 (Уленбек [10]). Пусть $E: M \times \mathbb{C}^{*} \rightarrow G^{\mathbb{C}}-$ семейство голоморфных сечений расслоения $\varphi^{-1}\left(T^{\mathbb{C}} G\right)$, наделенного канонической комплексной КМ-структурой, которое голоморфно зависит от $\lambda \in \mathbb{C}^{*}$. Предположим, что $E_{1}=$ е и выражсения

$$
\frac{1}{1-\lambda} E_{\lambda}^{-1} \bar{\partial} E_{\lambda} \quad u \quad \frac{1}{1-\lambda^{-1}} E_{\lambda}^{-1} \partial E_{\lambda}
$$

не зависят от $\lambda$. Тогда отображсение $\varphi:=E_{-1}$ гармонично.

ДокАЗАТЕльство. Утверждение достаточно проверить в произвольной локальной карте на $M$ с локальной координатой $z$. Обозначим

$$
A_{z}:=\frac{E_{\lambda}^{-1} \partial E_{\lambda}}{1-\lambda^{-1}}, \quad A_{\bar{z}}:=\frac{E_{\lambda}^{-1} \bar{\partial} E_{\lambda}}{1-\lambda}
$$

и положим $\alpha:=A_{z} d z+A_{\bar{z}} d \bar{z}$. По условию форма $\alpha$ не зависит от $\lambda$ и (при $\left.\lambda=-1\right)$ совпадает с $\frac{1}{2} \varphi^{-1} d \varphi$. Сечения $E_{\lambda}$ при любом $\lambda \in \mathbb{C}^{*}$ удовлетворяют системе уравнений

$$
\bar{\partial} E_{\lambda}=(1-\lambda) E_{\lambda} A_{\bar{z}}, \quad \partial E_{\lambda}=\left(1-\lambda^{-1}\right) E_{\lambda} A_{z} .
$$

Следовательно, для этой системы выполняется условие совместности, т.е. условие нулевой кривизны $(1.9)$ для связности $\alpha_{\lambda}=\frac{1}{2}\left(1-\lambda^{-1}\right) A_{z} d z+\frac{1}{2}(1-\lambda) A_{\bar{z}} d \bar{z}$. Отсюда вытекает в силу леммы 1.1, что отображение $\varphi$ гармонично. Предложение доказано.

Представление условия гармоничности в виде уравнения нулевой кривизны позволяет ожидать, что напространстве гармонических отображений $\varphi: M \rightarrow G$ действует преобразование типа Бэклунда, позволяющее "размножать" гармонические отображения. Такое преобразование в случае унитарной группы $G=\mathrm{U}(N)$ было найдено Уленбек [10]. Мы приведем его конструкцию в изложении Валли [11]. (Обобщение конструкции Уленбек на произвольные компактные группы Ли $G$ дано в [3], см. также [6].)

Обозначим через $\operatorname{Gr}\left(\mathbb{C}^{N}\right)$ полное грассманово многообразие

$$
\operatorname{Gr}\left(\mathbb{C}^{N}\right)=\bigcup_{r=0}^{N} G_{r}\left(\mathbb{C}^{N}\right)
$$


Точки $\operatorname{Gr}\left(\mathbb{C}^{N}\right)$, т.е. подпространства в $\mathbb{C}^{N}$, можно отождествить с эрмитовыми проекторами в $\mathbb{C}^{N}$, сопоставляя проектору $P$ его образ $\operatorname{Im} P \in \operatorname{Gr}\left(\mathbb{C}^{N}\right)$. С учетом этого многообразие $\operatorname{Gr}\left(\mathbb{C}^{n}\right)$ можно вложить в группу $\mathrm{U}(N)$ посредством

$$
\operatorname{Gr}\left(\mathbb{C}^{N}\right) \ni P \longmapsto P-P^{\perp}=V \in \mathrm{U}(N) .
$$

Образ $\operatorname{Gr}\left(\mathbb{C}^{N}\right)$ при этом вложении совпадает с подмножеством $\left\{V \in \mathrm{U}(N) \mid V^{2}=I\right\}$.

Тем самым, любое гладкое отображение $M \rightarrow \operatorname{Gr}\left(\mathbb{C}^{N}\right)$ можно рассматривать как семейство проекторов $\{P\}$ на $M$, которое в композиции с вложением $\operatorname{Gr}\left(\mathbb{C}^{N}\right) \hookrightarrow \mathrm{U}(N)$ порождает отображение $\varphi: M \rightarrow \mathrm{U}(N), \varphi=P-P^{\perp}$. Семейству проекторов $\left\{P^{\perp}\right\}$ на $M$ будет отвечать отображение $-\varphi: M \rightarrow \mathrm{U}(N)$. Отображение $\varphi=P-P^{\perp}$ голоморфно тогда и только тогда, когда $P^{\perp} \cdot \bar{\partial} P=0$.

Справедлива следуюшая теорема.

Теорема 1.4 (Уленбек [10], Валли [11]). Для любого гармонического отображения $\varphi: \mathbb{C P}^{1} \rightarrow \mathrm{U}(N)$ существует конечная последовательность $\varphi_{0}, \ldots, \varphi_{k}$ гармонических отображсений $\mathbb{C P}^{1} \rightarrow \mathrm{U}(N)$, удовлетворяющих условиям:

$$
\varphi_{0}=\text { const }, \quad \varphi_{k}=\varphi, \quad \varphi_{j}=\varphi_{j-1} \cdot\left(P_{j}-P_{j}^{\perp}\right), \quad 1 \leqslant j \leqslant k,
$$

и доставляющих каноническую факторизацию отображения $\varphi$ вида

$$
\varphi=\varphi_{0} \cdot\left(P_{1}-P_{1}^{\perp}\right) \cdots\left(P_{k}-P_{k}^{\perp}\right) .
$$

Отображение вида $\varphi \mapsto \varphi \cdot\left(P-P^{\perp}\right)$ Уленбек назьвает добавлением унитона. Таким образом, отображение $\varphi$ в теореме 1.4 получается из $\varphi_{0}=$ const добавлением $k$ унитонов. Можно показать, что в этом случае энергия отображения $\varphi$ равна $4 \pi k$.

\section{§ 2. Пространства петель и флаговые многообразия}

Конструкция Уленбек, изложенная в предыдущем параграфе, допускает естественную интерпретацию в терминах твисторных расслоений. Мы объясним это в п. 3.2 , но предварительно нам необходимо ввести основные понятия, относяшиеся к пространствам петель компактных групп Ли и их флаговым многообразиям.

2.1. Пространство петель компактной группы Ли. Для компактнойгрупш Ли $G$ обозначим через $L G=\operatorname{Map}\left(S^{1}, G\right)$ ее группу петель, т.е. пространство $C^{\infty}$-гладких отображений $S^{1} \rightarrow G$, где $S^{1}$ отождествляется с единичной окружностью в $\mathbb{C}$. Это группа Ли-Фреше относительно операции поточечного умножения (см. [9]). Пространством петель $\Omega G$ групы $G$ (или пространством центрированных петель) называется однородное пространство (правых смежных классов) группы $L G$ вида

$$
\Omega G=L G / G,
$$

где группа $G$ в знаменателе отождествляется с подгрупой постоянных отображений $S^{1} \rightarrow g_{0} \in G$.

Группа петель $L G$ действует на $\Omega G$ левыми сдвигами. Обозначим через $o$ начало (нейтральный элемент) в $\Omega G$, отождествляемое с классом постоянных отображений: $o:=[G]$. Пространство петель $\Omega G$ можно отождествить (как однородное пространство) с подгруппй $L_{1} G$ центрированных отображений в $L G$, переводящих $1 \in S^{1}$ в 
единицу $е$ групшы $G$. Касательное пространство к $\Omega G$ в начале $o$ отождествляется с пространством

$$
\Omega \mathfrak{g}=L \mathfrak{g} / \mathfrak{g},
$$

где $\mathfrak{g}$ - алгебра Ли группы $G, L \mathfrak{g}=\operatorname{Map}\left(S^{1}, \mathfrak{g}\right)$. Векторы касательного пространства $T_{o}(\Omega G)$ удобно задавать рядами Фурье: произвольный вектор $\xi$ комплексифицированного касательного пространства $T_{o}^{\mathbb{C}}(\Omega G)=T_{o}(\Omega G) \otimes \mathbb{C}$ имеет разложение Фурье вида $\xi=\sum_{k \neq 0} \xi_{k} e^{i k \theta}$, где коэффициенты $\xi_{k}$ принадлежат комплексифицированной алгебре Ли $\mathfrak{g}^{\mathbb{C}}$. Вектор $\xi \in T_{o}(\Omega G) \Longleftrightarrow \xi_{-k}=\bar{\xi}_{k}$.

Пространство петель $\Omega G$ обладает естественной симплектической структурой, инвариантной относительно действия группы петель $L G$ на $\Omega G$. Ввиду инвариантности, достаточно задать ее ограничение на $T_{o}(\Omega G)=\Omega \mathfrak{g}$. Для этого зафиксируем на алгебре Ли $\mathfrak{g}$ инвариантное скалярное произведение $\langle\cdot, \cdot\rangle$ и рассмотрим на $L \mathfrak{g} 2$-форму $\omega$ вида

$$
\omega(\xi, \eta)=\frac{1}{2 \pi} \int_{0}^{2 \pi}\left\langle\xi(\theta), \eta^{\prime}(\theta)\right\rangle d \theta, \quad \xi, \eta \in L \mathfrak{g} .
$$

Эта формула задает левоинвариантную замкнутую 2-форму на $L G$, причем $\omega(\xi, \eta)=0$, если хотя бы одно из отображений $\xi, \eta$ постоянно. Поэтому она спускается до левоинвариантной замкнутой 2 -формы на $\Omega \mathfrak{g}$, которая, как нетрудно проверить, невырождена и потому порождает симплектическую структуру на $\Omega G$. Более того, в случае полупростой групшы Ли $G$ она однозначно определяется выбором инвариантного скалярного произведения на $\mathfrak{g}$ (см. [8], а также [9]).

Инвариантная комплексная структура на $\Omega G$ обеспечивается "комплексным" представлением $\Omega G=L G / G$ в виде однородного пространства комплексной группы ЛиФреше $L G^{\mathbb{C}}=\operatorname{Map}\left(S^{1}, G^{\mathbb{C}}\right)$, где $G^{\mathbb{C}}$ - комплексификация групшы Ли $G$. Указанное представление имеет вид (см. [8], а также [9])

$$
\Omega G=L G^{\mathbb{C}} / L^{+} G^{\mathbb{C}},
$$

где $L^{+} G^{\mathbb{C}}=\operatorname{Hol}\left(\Delta, G^{\mathbb{C}}\right)$ есть подгруппа $L G^{\mathbb{C}}$, состоящая из отображений $S^{1} \rightarrow G^{\mathbb{C}}$, продолжаюшихся до голоморфных отображений круга $\Delta:=\{|z|<1\} \rightarrow G^{\mathbb{C}}$. Заметим, что в случае унитарной группы $G=\mathrm{U}(N)$ равенство $(2.2)$ означает, иными словами, что любая гладкая невырожденная матричная функция $T(\theta)$ на окружности допускает факторизацию вида

$$
T(\theta)=U(\theta) \cdot H_{+}(\theta),
$$

где $U(\theta)$ - гладкая унитарная (т.е. со значениями в $\mathrm{U}(N))$ матричная функция, а $H_{+}(\theta)$ продолжается до голоморфной (и гладкой вплоть до границы) невырожденной матричной функции в единичном круге $\Delta$. Указанная факторизация является параметрическим аналогом стандартного представления невырожденной матрицы в виде произведения унитарной и верхнетреугольной матрищ.

Индуцированную комплексньм представлением (2.2) инвариантную комплексную структуру $J^{1}$ на $\Omega G$ нетрудно описать и в терминах разложений Фурье. А именно, ограничение $J^{1}$ на комплексифицированное касательное пространство $T_{o}^{\mathbb{C}}(\Omega G)=\Omega \mathfrak{g}^{\mathbb{C}}$ в начале задается следуюшей явной формулой:

$$
\xi=\sum_{k \neq 0} \xi_{k} e^{i k \theta} \longmapsto J^{1} \xi=-i \sum_{k>0} \xi_{k} e^{i k \theta}+i \sum_{k<0} \xi_{k} e^{i k \theta} .
$$


Введенные симплектическая и комплексная структуры на $\Omega G$ согласованы в том смысле, что $\omega\left(J^{1} \xi, J^{1} \eta\right)=\omega(\xi, \eta)$ для всех $\xi, \eta \in T_{o}(\Omega G)$ и симметричная форма

$$
g^{1}(\xi, \eta):=\omega\left(\xi, J^{1} \eta\right) \text { на } T_{o}(\Omega G) \times T_{o}(\Omega G)
$$

положительно определена. Тем самым, ввиду инвариантности $\omega$ и $J^{1}$ эта форма продолжается до инвариантной римановой метрики $g^{1}$ на $\Omega G$. Иначе говоря, пространство петель $\Omega G$ является кәлеровым многообразием Фреше, наделенныц кәлеровой метрикой $g^{1}$.

Существование двух типов представлений пространства петель $\Omega G$ в виде однородного пространства вешественной и комплексной групп Ли-Фреше

$$
\Omega G=L G / G=L G^{\mathbb{C}} / L^{+} G^{\mathbb{C}}
$$

является характеристическим свойством пространства $\Omega G$. Конечномерные кэлеровы многообразия, обладаюшие аналогичным свойством, называются флаговыми многообразиями.

2.2. Флаговые многообразия. Приведем основные определения, относяшиеся к флаговым многообразиям (более подробно об этих многообразиях см. в [9]).

Для любого разложения натурального числа $N$ в сумму натуральных чисел $N=$ $r_{1}+\cdots+r_{n}$ можно построить многообразие флагов типа $\mathbf{r}:=\left(r_{1}, \ldots, r_{n}\right)$ в $\mathbb{C}^{N}$, полагая

$$
\begin{gathered}
\mathscr{F}_{\mathbf{r}}\left(\mathbb{C}^{N}\right)=\left\{\mathscr{V}=\left(V_{1}, \ldots, V_{n}\right): V_{i}-\text { линейные подпространства в } \mathbb{C}^{N}\right. \\
\text { такие, что } \left.V_{1} \subset \cdots \subset V_{n} \text { и } \operatorname{dim} V_{i}=r_{1}+\cdots+r_{i}\right\} .
\end{gathered}
$$

В частности, при $\mathbf{r}=(r, N-r)$ флаговое многообразие

$$
\mathscr{F}(r, N-r)\left(\mathbb{C}^{N}\right)=\left\{\text { подпространства } V \subset \mathbb{C}^{N} \text { размерности } r\right\}=G_{r}\left(\mathbb{C}^{N}\right)
$$

совпадает с грассмановым многообразием $r$-мерных подпространств в $\mathbb{C}^{N}$. При $\mathbf{r}=$ $(1, \ldots, 1)$ многообразие $\mathscr{F}_{\mathbf{r}}\left(\mathbb{C}^{N}\right)=: \mathscr{F}\left(\mathbb{C}^{N}\right)$ назьвается многообразием полных фла208

$$
\mathscr{F}\left(\mathbb{C}^{N}\right)=\left\{V_{1} \subset V_{2} \subset \cdots \subset V_{N-1} \subset V_{N}=\mathbb{C}^{N}: \operatorname{dim} V_{i}=i\right\} .
$$

Возврашаясь к определению флагового многообразия $\mathscr{F}_{\mathbf{r}}\left(\mathbb{C}^{N}\right)$, введем для $i=$ $1, \ldots, n$ подпространства $E_{i}:=V_{i-1}^{\perp} \cap V_{i}$, совпадающие с ортогональным дополнением $V_{i-1}$ в $V_{i}$ (относительно стандартного эрмитова скалярного произведения в $\mathbb{C}^{N}$ ). Тогда набор подпространств $\mathscr{E}=\left(E_{1}, \ldots, E_{n}\right)$ будет задавать разложение $\mathbb{C}^{N}$ в ортогональную прямую сумму подпространств $E_{i}$ размерности $r_{i}, i=1, \ldots, n$. Чтобы восстановить исходный флаг $\mathscr{V}=\left(V_{1}, \ldots, V_{n}\right)$ по набору $\mathscr{E}=\left(E_{1}, \ldots, E_{n}\right)$ указанного вида, достаточно положить: $V_{i}:=E_{1}+\cdots+E_{i}$ для $i=1, \ldots, n$. Тем самым, многообразие флагов $\mathscr{F}_{\mathbf{r}}\left(\mathbb{C}^{N}\right)$ можно эквивалентным образом определить как множество наборов $\mathscr{E}=\left(E_{1}, \ldots, E_{n}\right)$ попарно ортогональных подпространств $E_{i}$ размерности $r_{i}, i=1, \ldots, n$, задающих разложсение $\mathbb{C}^{N}$ в ортогональную прямую сум⿻м

$$
\mathbb{C}^{N}=\bigoplus_{i=1}^{n} E_{i}
$$


На многообразии флагов $\mathscr{F}_{\mathbf{r}}\left(\mathbb{C}^{N}\right)$ транзитивно действует унитарная группа $\mathrm{U}(N)$, так что $\mathscr{F}_{\mathbf{r}}\left(\mathbb{C}^{N}\right)$ является однородным пространством этой группы, а именно

$$
\mathscr{F}_{\mathbf{r}}\left(\mathbb{C}^{N}\right)=\mathrm{U}(N) / \mathrm{U}_{\mathbf{r}}(N)=\mathrm{U}(N) / \mathrm{U}\left(r_{1}\right) \times \cdots \times \mathrm{U}\left(r_{n}\right) .
$$

$\mathrm{C}$ другой стороны, группа $\mathrm{GL}(N, \mathbb{C})$ также действует на $\mathscr{F}_{\mathbf{r}}\left(\mathbb{C}^{N}\right)$ транзитивно, при этом подгруппа изотропии в начале будет совпадать с подгруппой $P_{\mathbf{r}}$ блочных верхнетреугольных матриц вида

$$
\left(\begin{array}{ccccc}
r_{1} & * & * & \ldots & * \\
0 & r_{2} & * & \ldots & * \\
\vdots & & \ddots & & \vdots \\
& & & & \\
0 & 0 & 0 & \ldots & r_{n}
\end{array}\right)
$$

Итак, наряду с “вещественньм" представлением (2.3) мы получаем для $\mathscr{F}_{\mathbf{r}}\left(\mathbb{C}^{N}\right)$ “комплексное” представление в виде однородного пространства группы $\operatorname{GL}(N, \mathbb{C})$

$$
\mathscr{F}_{\mathbf{r}}\left(\mathbb{C}^{N}\right)=\operatorname{GL}(N, \mathbb{C}) / P_{\mathbf{r}}
$$

В частных случаях $\mathbf{r}=(r, N-r)$ и $\mathbf{r}=(1, \ldots, 1)$ возникают известные представления в виде однородных пространств для грассманова многообразия

$$
G_{r}\left(\mathbb{C}^{N}\right)=\mathrm{U}(N) / \mathrm{U}(r) \times \mathrm{U}(N-r)=\mathrm{GL}(N, \mathbb{C}) / P_{(r, N-r)}
$$

и многообразия полных флагов

$$
\mathscr{F}\left(\mathbb{C}^{N}\right)=\mathrm{U}(N) / T^{N}=\mathrm{GL}(N, \mathbb{C}) / B_{+},
$$

где $T^{N}=\mathrm{U}(1) \times \cdots \times \mathrm{U}(1)$ есть $N$-мерный тор, а $B_{+}=P_{(1, \ldots, 1)}-$ стандартная борелевская подгрупп верхнетреугольных матриц.

Можно представить флаговое многообразие $\mathscr{F}_{\mathbf{r}}\left(\mathbb{C}^{N}\right)$ и в виде однородного пространства комплексной полупростой групшы, заменив группу $\operatorname{GL}(N, \mathbb{C})$ на $\operatorname{SL}(N, \mathbb{C})$.

Приведем общее алгебраическое определение флаговых многообразий. Для этого рассмотрим комплексную полупростую группу Ли $G^{\mathbb{C}}$ с алгеброй Ли $\mathfrak{g}^{\mathbb{C}}$. Фиксируем картановскую подалгебру $\mathfrak{h}^{\mathbb{C}}$ в $\mathfrak{g}^{\mathbb{C}}$ и корневое разложение алгебры $\mathfrak{g}^{\mathbb{C}}$ относительно этой картановской подалгебры:

$$
\mathfrak{g}^{\mathbb{C}}=\mathfrak{h}^{\mathbb{C}} \oplus \bigoplus_{\alpha \in \Delta} \mathfrak{g}_{\alpha}
$$

где $\Delta$ есть множество всех ненулевых корней алгебры $\mathfrak{g}^{\mathbb{C}}$ относительно $\mathfrak{h}^{\mathbb{C}}$. Зафиксируем в множестве $\Delta$ некоторое подмножество $\Pi=\left\{\alpha_{1}, \ldots, \alpha_{l}\right\}$ простых корней, выбор которого задает на множестве $\Delta$ отношение частичной упорядоченности, и обозначим через $\Delta^{+}$множество положительных корней относительно этого отношения. Тем самым, $\alpha \in \Delta^{+}$, если и только если $\alpha=\sum_{i=1}^{l} a_{i} \alpha_{i}$ с $a_{i}>0$. 
Введем теперь, по аналогии с борелевской подалгеброй верхнетреугольных матриц, стандартную борелевскую подалгебру $\mathfrak{b}_{+}$алгебры $\mathfrak{g}^{\mathbb{C}}: \mathfrak{b}_{+}=\mathfrak{h}^{\mathbb{C}} \oplus \mathfrak{n}_{+}$, где $\mathfrak{n}_{+}-$нильпотентная подалгебра $\mathfrak{g}^{\mathbb{C}}$ вида

$$
\mathfrak{n}_{+}=\bigoplus_{\alpha \in \Delta^{+}} \mathfrak{g}_{\alpha}
$$

$\mathrm{B}$ частном случае алгебры $\mathfrak{g}^{\mathbb{C}}=\operatorname{sl}(n, \mathbb{C})$ нильпотентная подалгебра $\mathfrak{n}_{+}$отождествляется с подалгеброй наддиагональных матрищ, a $\mathfrak{b}_{+}-$с подалгеброй верхнетреугольных матриц.

Вообще, будем назьвать борелевской подалгеброй любую подалгебру $\mathfrak{b}$ в $\mathfrak{g}^{\mathbb{C}}$, cопряженную стандартной борелевской подалгебре $\mathfrak{b}_{+}$относительно присоединенного действия группы $G^{\mathbb{C}}$ на $\mathfrak{g}^{\mathbb{C}}$. Любая подалгебра $\mathfrak{p}$ в $\mathfrak{g}^{\mathbb{C}}$, содержашая борелевскую подалгебру $\mathfrak{b}$, назьвается параболической. По аналогии с борелевскими параболические подалгебры $\mathfrak{p}$ можно также определить как подалгебры в $\mathfrak{g}^{\mathbb{C}}$, сопряженные одной из стандартных параболических подалгебр. Последние являются аналогами алгебр Ли $\mathfrak{p}_{\mathbf{r}}$ введенных выше параболических групп $P_{\mathbf{r}}$ и определяются следуюшим образом. Зафиксируем произвольное упорядоченное подмножество $\pi$ в множестве $\{1, \ldots, l\}$ и обозначим через $\Pi_{\pi} \subset \Pi$ множество простых корней алгебры $\mathfrak{g}^{\mathbb{C}}$ с номерами из $\pi$. Рассмотрим редуктивную подалгебру $\mathfrak{l}^{\mathbb{C}}$ вида

$$
\mathfrak{l}^{\mathbb{C}}=\mathfrak{h}^{\mathbb{C}} \oplus \bigoplus_{\alpha \in \Delta_{\pi}} \mathfrak{g}_{\alpha},
$$

где $\Delta_{\pi}$ - линейная оболочка простых корней $\Pi_{\pi}$ в $\Delta$, и нильпотентную подалгебру $\mathfrak{u}$ в $\mathfrak{g}^{\mathbb{C}}$, равную

$$
\mathfrak{u}=\bigoplus_{\alpha \in \Delta^{+} \backslash \Delta_{\pi}} \mathfrak{g}_{\alpha}
$$

Стандартная параболическая подалгебра $\mathfrak{p}_{\pi}$, отвечаюшая $\pi$, есть $\mathfrak{p}_{\pi}=\mathfrak{l}^{\mathbb{C}} \oplus \mathfrak{u}$. Она содержит стандартную борелевскую подалгебру $\mathfrak{b}_{+}$и потому является параболической. В случае алгебры Ли $\mathfrak{g}^{\mathbb{C}}=\operatorname{sl}(N, \mathbb{C})$ подалгебра $\mathfrak{l}^{\mathbb{C}}$ совпадает с подалгеброй блочно-диагональных матриц в $\operatorname{sl}(N, \mathbb{C})$, а $\mathfrak{u}-\mathrm{c}$ подалгеброй блочных наддиагональных матриц.

После этого алгебраического отступления вернемся к обшему определению флаговых многообразий комплексной полупростой групшы Ли $G^{\mathbb{C}}$. Пусть $\mathfrak{p}-$ произвольная параболическая подалгебра в $\mathfrak{g}^{\mathbb{C}}$ и $P$ - отвечающая ей параболическая подгруппа в $G^{\mathbb{C}}$. Флаговылм многообразием группы $G^{\mathbb{C}}$, отвечаюшим параболической подалгебре $\mathfrak{p}$, назьвается однородное пространство вида

$$
F=G^{\mathbb{C}} / P
$$

Наряду с “комплексным" представлением (2.6) флагового многообразия $F$ имеется и "вещественное" представление $F$ в виде однородного пространства вещественной групшы Ли. А именно, вспомним, что групша $G^{\mathbb{C}}$ является комплексификацией компактной групшы Ли $G$. Группа $G$ транзитивно действует на $G^{\mathbb{C}} / P$ и

$$
F=G / L
$$


где подгруппа $L=G \cap P$ в случае стандартной параболической подалгебры $\mathfrak{p}$ имеет своей алгеброй Ли вешественную форму $\mathfrak{l}$ введенной вьше подалгебры $\mathfrak{l}^{\mathbb{C}}$. Более инвариантно, подгрупу $L$ можно определить как централизатор $C(T)$ некоторого тора $T$ в группе $G$.

Итак, мы получаем для флагового многообразия $F$ вещественное и комплексное представления в виде однородного пространства

$$
F=G / L=G^{\mathbb{C}} / P \text {. }
$$

Из комплексного представления (2.6) вытекает, что $F$ - комплексное многообразие, которое обладает $G$-инвариантной комплексной структурой. Касательное пространство векторов типа $(1,0)$ в начале (относительно этой структуры) отождествляется с подалгеброй $\overline{\mathfrak{u}}$ из разложения

$$
\mathfrak{g}^{\mathbb{C}}=\mathfrak{l}^{\mathbb{C}} \oplus \mathfrak{u} \oplus \overline{\mathfrak{u}}, \quad \mathfrak{p}=\mathfrak{l}^{\mathbb{C}} \oplus \mathfrak{u} .
$$

Из вешественного представления (2.7) вытекают компактность и кэлеровость $F$.

Пространство петель $\Omega G$ можно рассматривать как универсальное флаговое многообразие группы $G$ в том смысле, что все флаговые многообразия $F$ группы $G$ допускают каноническую иммерсию в пространство петель $\Omega G$.

Для того чтобы доказать это, заметим, прежде всего, что вешественное представление многообразия флагов $F=G / L$ можно интерпретировать по-другому как представление $F$ в виде орбиты присоединенного действия Аd групшы $G$ на алгебре Ли $\mathfrak{g}$. Именно, орбита элемента $\xi \in \mathfrak{g}$ относительно присоединенного действия имеет вид $G / G(\xi)$, где подгруппа изотропии $G(\xi)$ в точке $\xi$ совпадает с централизатором $\xi$, равным $G(\xi)=\{g \in G: \operatorname{Ad} g(\xi)=\xi\}$. Все такие орбиты являются многообразиями флагов, и, наоборот, любое многообразие флагов компактной полупростой группы Ли можно представить в этом виде.

Рассмотрим теперь естественное действие ожружности $S^{1}$ на пространстве петель $\Omega G$ (отождествляемом с подгруппой $L_{1} G$ в группе петель $L G$ ), задаваемое врашением петель

$$
\lambda \cdot \gamma(z)=\gamma(\lambda)^{-1} \gamma(\lambda z), \quad \lambda \in S^{1},
$$

где $\gamma \in \Omega G$. Петля $\gamma$ является неподвижной точкой относительно $S^{1}$-действия тогда и только тогда, когда $\gamma(\lambda z)=\gamma(\lambda) \gamma(z)$ для всех $\lambda, z \in S^{1}$. Иначе говоря, $\gamma$ есть гомоморфизм $S^{1} \rightarrow G$. Но если $\gamma: S^{1} \rightarrow G$ - гомоморфизм, то и все сопряженные к $\gamma$ петли $\gamma_{g}=g \gamma g^{-1}$ для $g \in G$ также являются гомоморфизмами. Множество всех таких петель (классы сопряженности гомоморфизма $\gamma$ ) параметризуется точками однородного пространства $F_{\gamma}=G / G(\gamma)$, где $G(\gamma)$ - централизатор однопараметрической подгруппы $\gamma\left(S^{1}\right)$ в $G$. Указанное однородное пространство $F_{\gamma}$ отождествляется, как мы видели, с многообразием флагов групшы $G$. Итак, множество неподвижных точек $S^{1}$-действия на $\Omega G$ является несвязным облединением

$$
\operatorname{Fix}\left(S^{1}\right)=\bigcup_{\gamma} F_{\gamma}
$$

флаговых многообразий $F_{\gamma}$, где $\gamma$ пробегает множество классов сопряженности гомоморфизмов $S^{1} \rightarrow G$, а флаговые многообразия $F_{\gamma}$ вкладываются в $\Omega G$ в виде конечномерных кэлеровых подмногообразий. Тем самьм, построены отображения $\Gamma: F \rightarrow \Omega G$, которые, как можно показать, являются вполне геодезическими иммерсиями. 


\section{§3. Твисторные конструкции гармонических отображений}

Пусть $N$ - риманово многообразие и $Z$ - почти эрмитово многообразие, т.е. почти комплексное многообразие, наделенное эрмитовой метрикой. Расслоение $\pi: Z \rightarrow N$ называется твисторнылм, если для любого почти голоморфного отображения $\psi: M \rightarrow Z$ римановой поверхности $M$ в многообразие $Z$ его проекция $\pi \circ \psi$ является гармоническим отображением. Наличие твисторного расслоения у заданного риманова многообразия $N$ дает эффективньй способ построения гармонических отображений в $N$ как проекций почти голоморфных отображений в твисторное пространство $Z$ над $N$. Обшая теория твисторных расслоений рассматривается в [6], в данной статье мы ограничиваемся несколькими наиболее важными примерами, связанными с приложениями к интегрируемьм системам.

3.1. Гармонические отображения в грассмановы многообразия и симметрические пространства. Имеется целая серия твисторных расслоений над грассмановыми многообразиями $G_{r}\left(\mathbb{C}^{N}\right)$, задаваемых флаговыми многобразиями. Пусть $\mathscr{F}=\mathscr{F}_{\mathbf{r}}\left(\mathbb{C}^{N}\right)$ есть флаговое многообразие типа $\mathbf{r}=\left(r_{1}, \ldots, r_{n}\right)$ в $\mathbb{C}^{N}(\mathrm{~cm}$. п. 2.2), допускающее однородное представление (2.3):

$$
\mathscr{F}=\mathrm{U}(N) / \mathrm{U}\left(r_{1}\right) \times \cdots \times \mathrm{U}\left(r_{n}\right)
$$

На уровне алгебр Ли это представление соответствует разложению комплексифищированной алгебры Ли $\mathfrak{u}^{\mathbb{C}}(N)$ в прямую ортогональную сумму

$$
\begin{aligned}
\mathfrak{u}^{\mathbb{C}}(N) & \cong \mathfrak{g l}(N, \mathbb{C}) \cong \overline{\mathbb{C}^{N}} \otimes \mathbb{C}^{N} \\
& \cong\left(\bar{E}_{1} \oplus \cdots \oplus \bar{E}_{n}\right) \otimes\left(E_{1} \oplus \cdots \oplus E_{n}\right) \\
& \cong\left[\mathfrak{u}^{\mathbb{C}}\left(r_{1}\right) \oplus \cdots \oplus \mathfrak{u}^{\mathbb{C}}\left(r_{n}\right)\right] \oplus\left[\bigoplus_{i<j}\left(\bar{E}_{i} E_{j} \oplus \bar{E}_{j} E_{i}\right)\right]
\end{aligned}
$$

(В последней формуле опущен знак тензорного произведения в выражении $\bar{E}_{i} E_{j}$ и сопряженном к нему, чтобы сделать формулы обозримьми. Это правило применяется и в дальнейшем.)

Из приведенного разложения алгебры $\mathfrak{u}^{\mathbb{C}}(N)$ вытекает, что комплексифицированное касательное пространство $T_{o}^{\mathbb{C}} \mathscr{F}$ в начале $o \in \mathscr{F}$ есть

$$
T_{o}^{\mathbb{C}} \mathscr{F}=\bigoplus_{i<j} D_{i j}^{\mathbb{C}}:=\bigoplus_{i<j}\left(\bar{E}_{i} E_{j} \oplus \bar{E}_{j} E_{i}\right) .
$$

Каждую из компонент $D_{i j}$ можно наделить двумя различными комплексными структурами: для одной из них $(1,0)$-подпространство совпадает с $\bar{E}_{i} E_{j}$, для другой с $\bar{E}_{j} E_{i}$. По теореме Бореля-Хирцебруха [2] любая $U(N)$-инвариантная почти комплексная структура $J$ на $\mathscr{F}$ задается выбором одной из двух указанных комплексных структур на каждом $D_{i j}$. Будем называть канонической почти комплексную структуру $J^{1}$, для которой

$$
T_{o}^{1,0} \mathscr{F}=\bigoplus_{i<j} \bar{E}_{i} E_{j}
$$


Фиксируем упорядоченное подмножество $\sigma \subset\{1, \ldots, n\}$. Обозначим через $\sigma^{c}$ дополнение к $\sigma$ в $\{1, \ldots, n\}$ и положим $r=\sum_{i \in \sigma} r_{i}$. С каждьм таким подмножеством $\sigma$ ассоциировано однородное расслоение

$$
\pi=\pi_{\sigma}: \mathscr{F}=\frac{\mathrm{U}(N)}{\mathrm{U}\left(r_{1}\right) \times \cdots \times \mathrm{U}\left(r_{n}\right)} \longrightarrow \frac{\mathrm{U}(N)}{\mathrm{U}(r) \times \mathrm{U}(N-r)}=G_{r}\left(\mathbb{C}^{N}\right),
$$

сопоставляющее $\left(E_{1}, \ldots, E_{n}\right) \mapsto E=\bigoplus_{i \in \sigma} E_{i}$. Ему отвечает разложение комплексифицированного касательного расслоения $T^{\mathbb{C}} \mathscr{F}$ в прямую сумму вертикального и горизонтального подрасслоений. В частности, вертикальное подпространство в начале есть $\bigoplus_{i, j} D_{i j}^{\mathbb{C}}$, где $i<j$ и $i, j \in \sigma$, либо $i, j \in \sigma^{c}$. Соответственно, горизонтальное подпространство в начале совпадает с $\bigoplus_{i, j} D_{i j}^{\mathbb{C}}$, где $i<j$ и $i \in \sigma, j \in \sigma^{c}$ либо $i \in \sigma^{c}, j \in \sigma$.

Введем теперь, наряду с канонической, $\mathrm{U}(N)$-инвариантную почти комплексную структуру $J^{2}$ на $\mathscr{F}$, полагая $J^{2}=J^{1}$ на горизонтальных векторах и $J^{2}=-J^{1}$ на вертикальных векторах. Заметим, что построенное однородное расслоение $\pi_{\sigma}$, вообще говоря, не голоморфно относительно обеих почти комплексных структур. Кроме того, почти комплексная структура $J^{2}$ никогда не является интегрируемой. Однако оказьвается, что именно она связана с гармоническими отображениями, точнее, мы покажем далее, что расслоение $\pi_{\sigma}:\left(\mathscr{F}_{\mathbf{r}}\left(\mathbb{C}^{N}\right), J^{2}\right) \rightarrow G_{r}\left(\mathbb{C}^{N}\right)$ является твисторным.

Пусть $M$ - риманова поверхность. Обозначим через $\underline{\mathbb{C}}^{N}=M \times \mathbb{C}^{N}$ тривиальное расслоение над $M$, наделенное стандартной эрмитовой метрикой на слое $\mathbb{C}^{N}$. Любое подрасслоение $E \subset \underline{\mathbb{C}}^{N}$ ранга $r$ определяет отображение $\varphi_{E}: M \rightarrow G_{r}\left(\mathbb{C}^{N}\right)$ тавтологическим образом: $\varphi_{E}(p):=$ слой $E_{p}$ над точкой $p \in M$. Обратно, любое отображение $\varphi: M \rightarrow G_{r}\left(\mathbb{C}^{N}\right)$ задает подрасслоение $E \subset \underline{\mathbb{C}}^{N}$ ранга $r$.

Рассмотрим гладкое отображение римановой поверхности $M$ в грассманово многообразие $G_{r}\left(\mathbb{C}^{N}\right)$. Обозначим через $\pi$ и $\pi^{\perp}$ ортогональные проекции $\underline{\mathbb{C}}^{N}$ на подрасслоение $E$ и его ортогональное дополнение $E^{\perp}$. Расслоение $E$ наделяется комплексной КМ-структурой, которая в локальной карте на $M$ с локальной координатой $z$ задается $\bar{\partial}$-оператором $\partial_{E}^{\prime \prime}=\pi \circ \frac{\partial}{\partial z} \circ \pi$. Обратный образ $\varphi_{E}^{-1}\left(T^{\mathbb{C}} G_{r}\left(\mathbb{C}^{N}\right)\right)$ комплексифицированного касательного расслоения грассманиана при отображении $\varphi_{E}$ допускает разложение

$$
\varphi_{E}^{-1}\left(T^{\mathbb{C}} G_{r}\left(\mathbb{C}^{N}\right)\right) \cong \bar{E} E^{\perp} \oplus \overline{E^{\perp}} E,
$$

в терминах которого дифференциал $\varphi_{E}$ задается локально компонентами

$$
A_{E}^{\prime}:=\pi^{\perp} \circ \frac{\partial}{\partial z} \circ \pi, \quad A_{E}^{\prime \prime}:=\pi^{\perp} \circ \frac{\partial}{\partial \bar{z}} \circ \pi .
$$

(В дальнейшем мы иногда опускаем знак о для упрошения формул.) В частности, расслоение $E \subset \underline{\mathbb{C}}^{N}$ голоморфно тогда и только тогда, когда $A_{E}^{\prime \prime}=0$, и в этом случае комплексная КМ-структура на $E$ совпадает с индуцированной из $\underline{\mathbb{C}}^{N}$. Тогда

$$
\begin{aligned}
0 & =\pi^{\perp}\left[\frac{\partial}{\partial z}\left(\pi+\pi^{\perp}\right) \frac{\partial}{\partial \bar{z}}-\frac{\partial}{\partial \bar{z}}\left(\pi+\pi^{\perp}\right) \frac{\partial}{\partial z}\right] \pi \\
& =A_{E}^{\prime} \partial_{E}^{\prime \prime}+\partial_{E^{\perp}}^{\prime} A_{E}^{\prime \prime}-A_{E}^{\prime \prime} \partial_{E}^{\prime}-\partial_{E \perp}^{\prime \prime} A_{E}^{\prime}=A_{E}^{\prime} \partial_{E}^{\prime \prime}-\partial_{E \perp}^{\prime \prime} A_{E}^{\prime}
\end{aligned}
$$

Иначе говоря, расслоение $A_{E}^{\prime} \in \operatorname{Hom}\left(E, E^{\perp}\right)$ голоморфно относительно КМ-структур на $E$ и $E^{\perp}$. 
Вообще, будем называть подрасслоение $E \subset \underline{\mathbb{C}}^{N}$ гармоническим, если

$$
A_{E}^{\prime} \circ \partial_{E}^{\prime \prime}=\partial_{E \perp}^{\prime \prime} \circ A_{E}^{\prime}
$$

Гармоничность $E$ эквивалентна гармоничности отображения $\varphi_{E}: M \rightarrow G_{r}\left(\mathbb{C}^{N}\right)$ (см. [5]). Заметим также, что расслоение $E$ гармонично тогда и только тогда, когда гармонично его ортогональное дополнение $E^{\perp}$.

Более обшим образом, рассмотрим произвольньй набор $\mathscr{E}=\left(E_{1}, \ldots, E_{n}\right)$ попарно ортогональных подрасслоений $E_{i}$ в $\underline{\mathbb{C}}^{N}$ ранга $r_{i}$ с $r_{1}+\cdots+r_{n}=N$, порождающих разложение $\underline{\mathbb{C}}^{N}$ в ортогональную прямую сумму

$$
\underline{\mathbb{C}}^{N}=\bigoplus_{i=1}^{k} E_{i}
$$

Будем назьвать такой набор подрасслоений $\mathscr{E}=\left(E_{1}, \ldots, E_{n}\right)$ движущимся флагом на $M$. Он определяет, так же как и вьше, отображение $\psi_{\mathscr{E}}: M \rightarrow \mathscr{F} r_{1} \ldots r_{n}=\mathscr{F}$, сопоставляюшее точке $p \in M$ флаг, задаваемьй подпространствами $\left(E_{1, p}, \ldots, E_{n, p}\right)$. Обратно, любое гладкое отображение $\psi: M \rightarrow \mathscr{F}$ определяет движущийся флаг $\mathscr{E}=$ $\left(E_{1}, \ldots, E_{n}\right)$ указанного вьше вида, где $E_{i}=\psi^{-1} T_{i}$ есть обратньй образ естественного тавтологического расслоения $T_{i} \rightarrow \mathscr{F}:$ слой $T_{i}$ в точке $\mathscr{E} 0=\left(E_{1}^{0}, \ldots, E_{n}^{0}\right) \in \mathscr{F}$ совпадает, по определению, с подпространством $E_{i}^{0}$ для $1 \leqslant i \leqslant n$.

Так же как в грассмановом случае, дифференщиал $\psi_{\mathscr{E}}$ задается локально компонентами

$$
A_{i j}^{\prime}=\pi_{i} \circ \frac{\partial}{\partial z} \circ \pi_{j}, \quad A_{i j}^{\prime \prime}=\pi_{i} \circ \frac{\partial}{\partial \bar{z}} \circ \pi_{j}
$$

где $\pi_{i}: \underline{\mathbb{C}}^{N} \rightarrow E_{i}$ - ортогональная проекция.

Теорема 3.1 (Берстол-Саламон [4]). Построенное выше однородное расслоение $\pi_{\sigma}:\left(\mathscr{F}_{\mathbf{r}}\left(\mathbb{C}^{N}\right), J^{2}\right) \rightarrow G_{r}\left(\mathbb{C}^{N}\right)$ является твисторным, т.е. для любого $J^{2}-$ голоморфного отображения $\psi: M \rightarrow \mathscr{F}$ его проекция $\varphi:=\pi_{\sigma} \circ \psi: M \rightarrow G_{r}\left(\mathbb{C}^{N}\right)$ есть гармоническое отображение.

ДокАЗАТЕЛЬСтво. Достаточно показать, что для любого движущегося флага $\mathscr{E}=\left(E_{1}, \ldots, E_{n}\right)$, которому отвечает $J^{2}$-голоморфное отображение $\psi_{\mathscr{E}}: M \rightarrow \mathscr{F}$, расслоение $E:=\bigoplus_{i \in \sigma} E_{i}$ гармонично. Голоморфность отображения $\psi_{\mathscr{E}}$ означает, что

$$
A_{i j}^{\prime}=0=A_{j i}^{\prime \prime}, \quad \text { если }\left\{\begin{array}{l}
i>j \text { и } i, j \in \sigma \text { или } i, j \in \sigma^{c}, \\
i<j \text { и } i \in \sigma, j \in \sigma^{c} \text { или } i \in \sigma^{c}, j \in \sigma .
\end{array}\right.
$$

Если $k<l$ и $k \in \sigma, l \in \sigma^{c}$, то, так же как в грассмановом случае, имеем

$$
\begin{aligned}
0 & =\pi_{l} \sum_{i}\left[\frac{\partial}{\partial \bar{z}} \pi_{i} \frac{\partial}{\partial z}-\frac{\partial}{\partial z} \pi_{i} \frac{\partial}{\partial \bar{z}}\right] \pi_{k}=\sum_{i}\left(A_{l i}^{\prime \prime} A_{i k}^{\prime}-A_{l i}^{\prime} A_{i k}^{\prime \prime}\right) \\
& =\sum_{i \in \sigma^{c}} A_{l i}^{\prime \prime} A_{i k}^{\prime}-\sum_{i \in \sigma} A_{l i}^{\prime} A_{i k}^{\prime \prime}=\left(\sum_{i \in \sigma^{c}} A_{l i}^{\prime \prime}\right)\left(\sum_{i \in \sigma^{c}} A_{i k}^{\prime}\right)-\left(\sum_{i \in \sigma} A_{l i}^{\prime}\right)\left(\sum_{i \in \sigma} A_{i k}^{\prime \prime}\right) \\
& =\pi_{l}\left(\partial_{E}^{\prime \prime} \circ A_{E}^{\prime}-A_{E}^{\prime} \circ \partial_{E}^{\prime \prime}\right) \pi_{k} .
\end{aligned}
$$


Аналогичные соотношения вьполняются также при $k>l$, откуда следует, что $A_{E}^{\prime} \circ \partial_{E}^{\prime \prime}=\partial_{E \perp}^{\prime \prime} \circ A_{E}^{\prime}$, т.е. расслоение $E$ гармонично. Теорема доказана.

В случае, когда $M$ есть риманова сфера $\mathbb{C P}^{1}$, можно доказать обрашение теоремы 3.1, позволяющее получить твисторное описание всех гармонических отображений $\mathbb{C P}^{1} \rightarrow G_{r}\left(\mathbb{C}^{N}\right)$.

Теорема 3.2 (Берстол, см. [4]). Любое гармоническое отображение $\mathbb{C P}^{1} \rightarrow$ $G_{r}\left(\mathbb{C}^{n}\right)$ является проекцией $J^{2}$-голоморфного отображсения $\mathbb{C P}^{1} \rightarrow \mathscr{F}$ в многообразие флагов $\mathscr{F}=\mathscr{F}_{\mathbf{r}}\left(\mathbb{C}^{N}\right)$ при отображсении $\pi_{\sigma}:\left(\mathscr{F}_{\mathbf{r}}\left(\mathbb{C}^{N}\right), J^{2}\right) \rightarrow G_{r}\left(\mathbb{C}^{N}\right)$.

Доказательство указанного обрашения основано на теореме Биркгофа-Гротендика о классификации голоморфных векторных расслоений над $\mathbb{C P}^{1}$.

Твисторная конструкция гармонических отображений в грассмановы многообразия, изложенная в предыдущем параграфе, допускает непосредственное обобщение на широкий класс симметрических пространств, яВляюшихся базами однородных расслоений флаговых многообразий. Это так называемые внутренние симметрические пространства, класс которых включает в себя, помимо грассмановых многообразий, четномерные сферы, эрмитовы симметрические пространства и т. д. (подробнее об этих пространствах см. в [3]). Приведем конструкцию внутренних симметрических пространств, ассоциированных с флаговыми многообразиями.

Пусть $F=G / L=G^{\mathbb{C}} / P$ есть флаговое многообразие компактной полупростой групшы Ли $G$. Алгебра Ли р параболической грушы $P$ представляется в виде

$$
\mathfrak{p}=\mathfrak{l}^{\mathbb{C}} \oplus \mathfrak{u},
$$

где $\mathfrak{l}^{\mathbb{C}}$ и $\mathfrak{u}$ - редуктивная и нильпотентная подалгебры $\mathfrak{p}$, построенные в п. 2.2 для стандартных параболических подалгебр. Здесь мы дадим другое определение этих подалгебр в терминах так называемого канонического элемента.

ПРЕДЛОЖЕНИЕ 3.3 (см. [3; теорема 4.4]). Для любой параболической подалгебры $\mathfrak{p}$ найдется единственный әлемент $\xi$ (принадлежсащий чентру алгебры $\left.\mathfrak{l}^{\mathbb{C}}\right)$, для которого все собственные значения оператора аd $\xi$ являются целыми мнимыми числами, т.е. принадлежат $\sqrt{-1} \mathbb{Z}$. Указанный әлемент $\xi$ называется каноническим элементом параболической подалгебры $\mathfrak{p}$.

Обозначим через $\mathfrak{g}_{j}$ собственное подпространство оператора $\mathrm{ad} \xi$ с собственным значением $\sqrt{-1} j$. В этих терминах подалгебры $\mathfrak{p}$ и $\mathfrak{u}$ задаются формулами

$$
\mathfrak{p}=\bigoplus_{i \geqslant 0} \mathfrak{g}_{i}, \quad \mathfrak{u}=\bigoplus_{i \geqslant 1} \mathfrak{g}_{i}
$$

Построим теперь симметрическое пространство $N=N(F)$, ассочиированное с флаговым многообразием $F$, полагая $N=G / K$, где $K$ - подгруппа $G$ с алгеброй Ли

$$
\mathfrak{k}=\mathfrak{g} \cap\left[\bigoplus_{i} \mathfrak{g}_{2 i}\right] .
$$

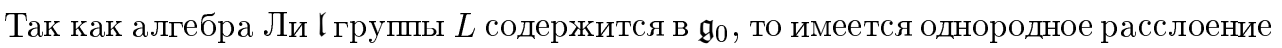

$$
F=G / L \longrightarrow G / K=N
$$


флагового многообразия $F$ над ассоциированным с ним симметрическим пространством $N$. Подгруппу $K$ можно также определить с помощью оператора $\tau_{\xi}:=\operatorname{Ad} \exp \pi \xi$ на $\mathfrak{g}$. Поскольку собственные значения $\mathrm{ad} \xi$ являются мнимыми цельми числами, оператор $\tau_{\xi}$ задает инволюцию на $\mathfrak{g}$, экспонента которой дает инволюцию $\sigma$ групшы $G$. Подгрупа $K$ совпадает со связной компонентой единицы $G_{0}^{\sigma}$ в группе неподвижных точек $G^{\sigma}$ этой инволюции.

Итак, мы построили для каждого флагового многообразия $F$ ассоциированное с ним симметрическое G-пространство $N=N(K)$ (которое определяется единственным образом) и каноническое однородное расслоение $F \rightarrow N$. Заметим, что расслоение $F \rightarrow N$ определяется, вообе говоря, неоднозначно, но весь произвол состоит в том, что различные точки $N$ могут иметь один и тот же стабилизатор $K$. Число таких точек конечно, поэтому имеется не более конечного числа расслоений указанного вида.

Определим, так же как и выше, почти комплексные структуры $J^{1}$ и $J^{2}$ на $F$. В терминах подалгебр $\mathfrak{g}_{j}$ они описываются следуюшим образом. Подпространство $(1,0)$ векторов $T_{o}^{1,0} F$ почти комплексной структуры $J^{1}$ в начале $o$ совпадает с

$$
\overline{\mathfrak{u}}=\bigoplus_{i<0} \mathfrak{g}_{i}
$$

тогда как подпространство $(1,0)$-векторов $T_{o}^{1,0} F$ почти комплексной структуры $J^{2}$ в начале $о$ есть

$$
\left[\bigoplus_{i<0} \mathfrak{g}_{2 i}\right] \oplus\left[\bigoplus_{i \geqslant 0} \mathfrak{g}_{2 i+1}\right] .
$$

В остальные точки $F$ эти структуры разносятся с помошюю действия групшы $G$ на $F$.

Справедлив следуюший аналог теоремы 3.1 .

ТЕОРема 3.4 (Берстол-Ронсли [3]). Любое из канонических расслоений $\pi:\left(F, J^{2}\right) \rightarrow N(F)$ является твисторным расслоением.

Иными словами, проекция $\varphi:=\pi \circ \psi$ любого $J^{2}$-голоморфного отображения $\psi: M \rightarrow F$ римановой поверхности $M$ в многообразие фолагов $F$ является гармоническим отображением $\varphi: M \rightarrow N$. Некоторые условия, достаточные для справедливости обратного утверждения, т.е. для существования твисторного поднятия $\psi: M \rightarrow F$ заданного гармонического отображения $\varphi: M \rightarrow N$, приведены в [3] (см. также [6]).

3.2. Пространство петель как твисторное расслоение. В этом пункте будет дана твисторная интерпретация конструкции Уленбек из п. 1.2.

Обозначим через

$$
\pi: \Omega G \rightarrow G, \quad \gamma \rightarrow \gamma(-1),
$$

отображение, сопоставляющее петле $\gamma$ ее значение в точке $-1 \in S^{1}$. Это отображение является аналогом однородного расслоения $\pi: F \rightarrow N$, рассмотренного в предыдущем пункте.

В п. 1.2 мы сопоставили гармоническому отображению $\varphi: M \rightarrow G$ из римановой поверхности $M$ в компактную группу Ли $G$ отображение $E_{\lambda}: M \rightarrow G^{\mathbb{C}}$, которое голоморфно зависит от $\lambda \in \mathbb{C}^{*}$ и удовлетворяет условиям

$$
E_{1}=e, \quad E_{-1}=\varphi, \quad E_{\lambda}\left(p_{0}\right)=e \text { для всех } \lambda \in \mathbb{C}^{*} .
$$


Рассматривая $E_{\lambda}$ при $\lambda \in S^{1}$, получим отображение $E: S^{1} \times M \rightarrow G$ или, иначе, отображение $E: M \rightarrow \Omega G$ в пространство петель $\Omega G$.

В п. 1.2 была построена почти комплексная структура $J^{1}$ на пространстве $\Omega G$. Теперь, так же как в предыдущем пункте, мы введем на $\Omega G$ почти комплексную структуру $J^{2}$, ответственную за гармонические отображения. А именно, для вектора $\xi=$ $\sum_{k \neq 0} \xi_{k} e^{i k \theta}$, принадлежашего касательному пространству $T_{o}^{\mathbb{C}}(\Omega G)=\Omega \mathfrak{g}^{\mathbb{C}}$, положим

$$
J^{2} \xi=-i \sum_{k \neq 0}(-1)^{k+1} \operatorname{sign} k \cdot \xi_{k} e^{i k \theta} .
$$

Структура $J^{2}$ совпадает с $J^{1}$ при нечетных $k$ и с $-J^{1}-$ при четных $k$.

Заметим, что построенное отображение $E: M \rightarrow \Omega G$ обладает тем свойством, что разложение Фурье формы $E_{\lambda}^{-1} d E_{\lambda}$ по $\lambda \in S^{1}$ содержит, согласно формуле (1.11), только члены, линейные по $\lambda$ и $\lambda^{-1}$. Назовем такое отображение $E: M \rightarrow \Omega G c y$ пергоризонтальныц.м. Тем самьм, отображение $E: M \rightarrow \Omega G$ супергоризонтально и потому голоморфно относительно обеих почти комплексных структур $J^{1}$ и $J^{2}$. Оно задает, очевидно, твисторное поднятие отображения $\varphi: M \rightarrow G$, которое мы будем называть каноническим. Итак, доказано следующее предложение.

ПреДЛОЖЕНИЕ 3.5. Пусть $\varphi: M \rightarrow G-$ иентрированное $\left(\right.$ m.е. $\left.\varphi\left(p_{0}\right)=e \in G\right)$ гармоническое отображсние. Тогда его каноническое твисторное поднятие $E: M \rightarrow \Omega G$ супергоризонтально и голоморфно относительно обеих почти комплексных структур $J^{1} u J^{2}$.

Предложение 1.3 из п. 1.2 показьвает, обратно, что проекция $E_{-1}=:$ л любого супергоризонтального голоморфного отображения $E: M \rightarrow \Omega G$ является гармоническим отображением. Последнее утверждение можно усилить следуюшим образом.

ПрЕДлОЖенИЕ 3.6 (Берстол). Отображсение $\pi:\left(\Omega G, J^{2}\right) \rightarrow G$ является твисторныцм расслоением, т.е. проекция $\varphi:=\pi \circ \psi$ любого $\mathrm{J}^{2}$-голоморфного отображения $\psi: M \rightarrow \Omega G$ римановой поверхности $M$ является гармоническим отображением.

Доказательство этого утверждения проводится так же, как в случае однородных флаговых расслоений $\pi: F \rightarrow N$ (см. теорему 3.4).

В п. 2.2 отмечалось, что пространство петель $\Omega G$ можно рассматривать как универсальное флаговое многообразие групшы $G$ в том смысле, что все флаговые многообразия $F$ группы $G$ вкладьваются в $\Omega G$. Точно так же твисторное расслоение $\pi: \Omega G \rightarrow G$ можно рассматривать как универсальное твисторное расслоение в следуюшем смысле.

Рассмотрим, как и в п. 2.2 , естественное $S^{1}$-действие на $\Omega G$, порождаемое вращением петель. Указанное действие индуцирует $S^{1}$-действие на отображениях $\Phi: M \rightarrow$ $\Omega G$, которое сохраняет $J^{1}$ - и $J^{2}$-голоморфность этих отображений. Справедливо следующее предложение.

ПРЕДЛОЖЕНИЕ 3.7 (УЛенбек [10]). Пусть отображение $\Phi: M \rightarrow \Omega G$, являющееся каноническим поднятием гармонического отображения $\varphi: M \rightarrow G$, неподвижно относительно $S^{1}$-действия: $S^{1} \cdot \Phi=\Phi$. Тогда образ $\Phi(M)$ содержится 
в некотором флаговом многообразии $F=G / L$, а образ $\varphi(M)$ - в симметрическом пространстве $N(F)=G / K$.

Тем самым, имеется коммутативная диаграмма

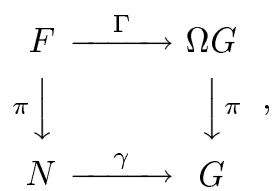

в которой $\pi: F \rightarrow N(F)$ есть твисторное расслоение над симметрическим пространством $N(F)$, построенное в п. 3.1, а отображение $\Gamma$ - вполне геодезическая иммерсия, построенная в п. 2.2, которая голоморфна относительно обеих почти комплексных структур $J^{1}$ и $J^{2}$.

Горизонтальные отображения в приведенной диаграмме допускают простое описание в терминах канонического элемента, введенного в п. 3.1. Именно, предположим, что группа $G$ имеет тривиальный центр, и рассмотрим флаговое многообразие $F=G / L=G^{\mathbb{C}} / P$ с каноническим элементом $\xi$. Условие тривиальности центра $G$ означает, что $\exp (2 \pi \xi)=e \in G$. Поэтому мы можем определить отображение $\Gamma: F \rightarrow \Omega G$, полагая его на нейтральном элементе $o \in F$ равным $\Gamma(o):=$ отображение $\left\{e^{i t} \mapsto \exp (t \xi)\right\}$ и распространяя в другие точки $G / L$ с помощью левых сдвигов группы $G$. С другой стороны, имеется естественное отображение $\gamma: N \rightarrow G$, сопоставляющее точке $x$ внутреннего симметрического пространства $N$, ассоциированного с $F$, элемент $\gamma(x)$ групшы $G$, порождаюший инволюцию в точке $x$. Оба отображения $\Gamma$ и $\gamma$ являются вполне геодезическими иммерсиями.

3.3. Гармонические отображения в гильбертов грассманиан и пространства петель. В этом пункте мы рассмотрим гармонические отображения в грассманиан комплексного гильбертова пространства. Данньй класс содержит, в частности, все гармонические отображения в пространства петель $\Omega G$, поскольку пространства $\Omega G$ допускают изометрическое вложение в гильбертов грассманиан.

Напомним основные определения, относящиеся к гильбертову грассманиану (более подробно об этом см. [8], [9]). Пусть $H$ есть комплексное (сепарабельное) гильбертово пространство, моделью которого для нас является пространство $L^{2}\left(S^{1}, \mathbb{C}\right)$ квадратично интегрируемых функций на окружности или его векторньй аналог $L^{2}\left(S^{1}, \mathbb{C}^{N}\right)$. Предположим, что $H$ обладает поляризаиией, т.е. разложением

$$
H=H_{+} \oplus H_{-}
$$

в прямую ортогональную сумму бесконечномерных замкнутых подпространств. В случае $H=L^{2}\left(S^{1}, \mathbb{C}^{N}\right)$ в качестве таких подпространств выступают

$$
\begin{aligned}
& H_{+}=\left\{f \in H: f(z)=\sum_{k \geqslant 0} f_{k} z^{k}, f_{k} \in \mathbb{C}^{N}\right\}, \\
& H_{-}=\left\{f \in H: f(z)=\sum_{k<0} f_{k} z^{k}, f_{k} \in \mathbb{C}^{N}\right\},
\end{aligned}
$$

где $z=e^{i \theta}$. 
Обозначим через $\mathrm{GL}(H)$ группу линейных ограниченных операторов на $H$, имеющих ограниченньй обратньй. Относительно поляризации (3.1) любой линейньй опеpaтop $A \in$ End $H$ записьвается в блочном виде

$$
A=\left(\begin{array}{ll}
a & b \\
c & d
\end{array}\right)=\left(\begin{array}{ll}
a: H_{+} \rightarrow H_{+}, & b: H_{-} \rightarrow H_{+} \\
c: H_{+} \rightarrow H_{-}, & d: H_{-} \rightarrow H_{-}
\end{array}\right)
$$

По определению, группа $\mathrm{GL}_{\mathrm{res}}(H)$ состоит из преобразований $A \in \mathrm{GL}(H)$, для которых "внедиагональные" члены $b$ и $c$ являются операторами Гильберта-Шмидта (коротко: HS-операторами). Иньми словами, группа $\mathrm{GL}_{\mathrm{res}}(H)$ состоит из преобразований $A \in \mathrm{GL}(H)$, в которых "внедиагональные" члены $b$ и с операторов $A$ из $\mathrm{GL}_{\mathrm{res}}(H)$ "малы” по сравнению с “диагональными” членами $a$ и $d$. Через $\mathrm{U}_{\text {res }}(H)$ обозначим пересечение $\mathrm{GL}_{\mathrm{res}}(H)$ с группой $\mathrm{U}(H)$ унитарных операторов в $H$.

Напомним, что линейный оператор $T: H_{1} \rightarrow H_{2}$, действующий из гильбертова пространства $H_{1}$ в гильбертово пространство $H_{2}$, назьвается оператором Гильбер-

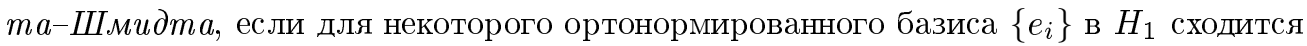
ряд $\sum_{i}\left\|T e_{i}\right\|<\infty$. Операторы Гильберта-Шмидта $T: H_{1} \rightarrow H_{2}$ образуют комплексное гильбертово пространство $\mathrm{HS}\left(H_{1}, H_{2}\right)$ с нормой, равной

$$
\|T\|_{2}=\left(\sum_{i=1}^{\infty}\left\|T e_{i}\right\|^{2}\right)^{1 / 2}
$$

Пространство $\mathrm{HS}(H, H)$ операторов Гильберта-Шмидта, действующих в гильбертовом пространстве $H$, является двусторонним идеалом в алгебре $\mathrm{L}(H)$ всех ограниченных линейных операторов в $H$.

С группой $\mathrm{GL}_{\mathrm{res}}(H)$, так же как в конечномерном случае, связано некоторое грассманово многообразие $\operatorname{Gr}_{\text {res }}(H)$, которое мы и назьваем гильбертовыл грассманианом. Более подробно, обозначим через $\mathrm{Gr}_{\mathrm{res}}(H)$ множество замкнутых подпространств $W \subset H$ таких, что ортогональная проекция $\mathrm{pr}_{+}: W \rightarrow H_{+}$является фредгольмовым оператором, а ортогональная проекция $\mathrm{pr}_{-}: W \rightarrow H_{-}$есть оператор Гильберта-Шмидта. Иначе говоря, множество $\mathrm{Gr}_{\mathrm{res}}(H)$ состоит из подпространств $W \subset H$, которые "мало" отличаются от подпространства $H_{+}$в том смысле, что проекция $\mathrm{pr}_{+}: W \rightarrow H_{+}$"близка" к изоморфизму, а проекция $\mathrm{pr}_{-}: W \rightarrow H_{-}$"мала". Эквивалентное определение: подпространство $W \in \mathrm{Gr}_{\mathrm{res}}(H)$, если оно является образом линейного оператора $w: H_{+} \rightarrow H$ такого, что оператор $w_{+}:=p_{+} \circ w$ фредгольмов, а $w_{-}:=\mathrm{pr}_{-}$ow- оператор Гильберта-Шмидта.

Легко видеть, что если $W \in \mathrm{Gr}_{\mathrm{res}}(H)$, то график любого HS-оператора $w^{\prime}: W \rightarrow$ $W^{\perp}$ также принадлежит $\operatorname{Gr}_{\text {res }}(H)$. Обозначим множество таких подпространств через $U_{W}$ :

$$
\begin{aligned}
U_{W} & =\left\{W^{\prime} \in \operatorname{Gr}_{\text {res }}(H): W^{\prime} \text { есть график HS-оператора } w^{\prime}: W \rightarrow W^{\perp}\right\} \\
& =\left\{W^{\prime} \in \operatorname{Gr}_{\text {res }}(H): \text { ортогональная проекция } W^{\prime} \rightarrow W \text { есть изоморфизм }\right\} .
\end{aligned}
$$

Открытые подмножества $\left\{U_{W}\right\}$ играют роль атласа координатных окрестностей для грассманова многообразия $\mathrm{Gr}_{\mathrm{res}}(H)$. 
Введенная вьше группа $\mathrm{GL}_{\text {res }}(H)$ естественньм образом действует на $\mathrm{Gr}_{\text {res }}(H)$. Это действие транзитивно, так же как и действие унитарной подгрупшы $\mathrm{U}_{\mathrm{res}}(H)$. Подгруппа изотропии $\mathrm{U}_{\text {res }}(H)$ в точке $H_{+} \in \mathrm{Gr}_{\text {res }}(H)$ совпадает с $\mathrm{U}\left(H_{+}\right) \times \mathrm{U}\left(H_{-}\right)$, тем самым грассманиан $\mathrm{Gr}_{\text {res }}(H)$ является однородньм пространством групшы $\mathrm{U}_{\text {res }}(H)$ вида

$$
\mathrm{Gr}_{\mathrm{res}}(H)=\mathrm{U}_{\mathrm{res}}(H) / \mathrm{U}\left(H_{+}\right) \times \mathrm{U}\left(H_{-}\right) .
$$

Пользуясь этим представлением, можно доказать следующее предложение.

ПреДлОЖение 3.8 (см. [8], а также [9]). Гильбертов грассманиан $\mathrm{Gr}_{\mathrm{res}}(H)$ является комплексным гильбертовым многообразием, локальная модель которого совпадает с пространством операторов Гильберта-Шмидта. Атлас на $\mathrm{Gr}_{\mathrm{res}}(H)$ задается координатными окрестностями

$$
U_{W}=\left\{W^{\prime} \in \operatorname{Gr}_{\text {res }}(H): W^{\prime} \text { eсть график HS-onepamopa } w^{\prime}: W \rightarrow W^{\perp}\right\},
$$

а карты - отображсениями $U_{W} \ni W^{\prime} \mapsto w^{\prime} \in \operatorname{HS}\left(W, W^{\perp}\right)$.

Многообразие $\mathrm{Gr}_{\text {res }}(H)$ имеет счетное число связных компонент, нумеруемых индексом фредгольмова оператора $w_{+}$для подпространства $W \in \mathrm{Gr}_{\text {res }}(H)$, совпадающего с образом линейного оператора $w: H_{+} \rightarrow H$. Будем говорить, что подпространство $W$ имеет виртуальную размерность $d$, если индекс $w_{+}$равен $d$. Обозначим компоненту $\mathrm{Gr}_{\mathrm{res}}(H)$, состояшую из подпространств $W$ виртуальной размерности $d$, через $\mathrm{G}_{d}(H)$. Тогда

$$
\operatorname{Gr}_{\mathrm{res}}(H)=\bigcup_{d} \mathrm{G}_{d}(H)
$$

С этим разложением связано другое "координатное" представление $\operatorname{Gr}_{\text {res }}(H)$, которое мы опишем в терминах модели $H$, задаваемой гильбертовым пространством $L^{2}\left(S^{1}, \mathbb{C}\right)$. Наделим $L^{2}\left(S^{1}, \mathbb{C}\right)$ каноническим базисом $\left\{z^{k}\right\}_{k \in \mathbb{Z}}$, так что подпространство $H_{+}$будет порождаться элементами $\left\{z^{k}\right\}_{k \in \mathbb{Z}_{+}}$, а $H_{-}$- элементами $\left\{z^{k}\right\}_{k \in \mathbb{Z}_{-}}$, где через $\mathbb{Z}_{+}$обозначено подмножество целых неотрицательных чисел в $\mathbb{Z}$, а через $\mathbb{Z}_{-}$его дополнение в $\mathbb{Z}$. Рассмотрим в качестве "координатных" подпространств в $H$ замкнутые линейные подпространства $H_{S}$, порождаемые векторами $\left\{z^{s}\right\}_{s \in S}$, которые нумеруются подмножествами $S \subset \mathbb{Z}$, сравнимыми $c \mathbb{Z}_{+}$. Иначе говоря, подмножествами $S$, для которых множества $S-\mathbb{Z}_{+}$и $\mathbb{Z}_{+}-S$ состоят из конечного числа точек. Число $\left|S-\mathbb{Z}_{+}\right|-\left|\mathbb{Z}_{+}-S\right|$ назьвается виртуальной мощностью $S$. Заметим, что виртуальная размерность подпространства $H_{S}$ в точности совпадает с виртуальной мощностью $S$.

Нетрудно показать, что для любого $W \in \mathrm{Gr}_{\text {res }}(H)$ найдется подмножество $S$, сравнимое с $\mathbb{Z}_{+}$, для которого ортогональная проекция $\operatorname{pr}_{S}: W \rightarrow H_{S}$ является изоморфизмом. Пользуясь этим свойством, введем на $\operatorname{Gr}_{\text {res }}(H)$ новый атлас, задаваемый открытыми множествами $\left\{U_{S}\right\}$, где $S$ - подмножество $\mathbb{Z}$, сравнимое с $\mathbb{Z}_{+}$. Координатная окрестность $U_{S}=U_{H_{S}}$ состоит, по определению, из подпространств, являюшихся графиками операторов Гильберта-Шмидта $H_{S} \rightarrow H_{S}^{\perp}=H_{S^{\perp}}$, где $S^{\perp}=\mathbb{Z}-S$.

Так как $\mathrm{U}_{\mathrm{res}}(H)$ транзитивно действует на грассманиане $\mathrm{Gr}_{\mathrm{res}}(H)$, то можно построить $\mathrm{U}_{\text {res }}(H)$-инвариантную кэлерову метрику на $\operatorname{Gr}_{\text {res }}(H)$ по скалярному произведению на касательном пространстве $T_{H_{+}} \mathrm{Gr}_{\text {res }}(H)$ в начале $H_{+} \in \mathrm{Gr}_{\text {res }}(H)$, инвариантному относительно действия подгрупшы изотропии $\mathrm{U}\left(H_{+}\right) \times \mathrm{U}\left(H_{-}\right)$. Касательное пространство $T_{H_{+}} \mathrm{Gr}_{\text {res }}(H)$ совпадает с пространством операторов Гильберта-Шмидта $\mathrm{HS}\left(H_{+}, H_{-}\right)$, а инвариантное скалярное произведение на нем задается 
формулой

$$
(A, B) \mapsto \operatorname{Re}\left\{\operatorname{tr}\left(A B^{*}\right)\right\}, \quad A, B \in \operatorname{HS}\left(H_{+}, H_{-}\right) .
$$

Заметим, что мнимая часть комплексного скалярного произведения $\operatorname{tr}\left(A B^{*}\right)$ определяет на $T_{H_{+}} \mathrm{Gr}_{\text {res }}(H)$ невырожденную инвариантную 2-форму, которая продолжается до $\mathrm{U}_{\mathrm{res}}(H)$-инвариантной симплектической структуры на многообразии $\mathrm{Gr}_{\mathrm{res}}(H)$. Тем самым, на $\mathrm{Gr}_{\mathrm{res}}(H)$ задана кэлерова структура, преврашающая его в кәлерово гильбертово многообразие.

Ввиду разложения (3.2) исследование гармонических отображений в $\mathrm{Gr}_{\mathrm{res}}(H)$ cводится к исследованию гармонических отображений в грассманианы $\mathrm{G}_{d}(H)$ виртуальной размерности $d$, которое можно проводить теми же методами, что и в случае грассманова многообразия $G_{r}\left(\mathbb{C}^{N}\right)$. Так же как в п. 2.2 , строятся флаговые многообразия $\mathscr{F}=\mathscr{F}_{\mathbf{r}}(H)$ типа $\mathbf{r}=\left(r_{1}, \ldots, r_{n}\right)$, состояшие из наборов $\mathscr{W}=\left(W_{1}, \ldots, W_{n}\right)$ подпространств $W_{i} \subset H$ виртуальной размерности $r_{i}$ с $r_{1}+\cdots+r_{n}=d$. Далее, для любого упорядоченного подмножества $\sigma \subset\{1, \ldots, d\}$ строится однородное расслоение $\pi_{\sigma}: \mathscr{F} \rightarrow G_{d}(H)$, сопоставляющее $\left(W_{1}, \ldots, W_{n}\right) \mapsto W=\bigoplus_{i \in \sigma} W_{i}$. Так же как в п. 3.1, на флаговом многообразии $\mathscr{F}$ вводятся почти комплексные структуры $J^{1}$ и $J^{2}$. Справедливо следующее предложение, которое доказьвается так же, как теорема 3.1 .

ПРЕДЛОЖЕНИЕ 3.9. Однородное расслоение $\pi_{\sigma}:\left(\mathscr{F}_{\mathbf{r}}(H), J^{2}\right) \rightarrow G_{d}(H)$ является твисторным, т.е. для любого $J^{2}$-голоморфного отображсения $\psi: M \rightarrow \mathscr{F}$ его проекция $\varphi:=\pi_{\sigma} \circ \psi: M \rightarrow G_{d}(H)$ есть гармоническое отображсение.

Обратимся теперь к гармоническим отображениям в пространства петель и построим, прежде всего, изометрическое вложение пространств $\Omega G$ в гильбертов грассманиан $\mathrm{Gr}_{\text {res }}(H)$, упомянутое в начале этого пункта.

Рассмотрим сначала группу петель $L G$, где $G=\operatorname{GL}(N, \mathbb{C})$ - группа невырожденных комплексных $(N \times N)$-матриц. Выберем в качестве комплексного гильбертова пространства $H$ пространство $L^{2}\left(S^{1}, \mathbb{C}^{N}\right)$ с естественной поляризацией, описанной в начале этого пункта. Каждой петле $\gamma \in \operatorname{LGL}(N, \mathbb{C})$ можно сопоставить линейньй оператор $M_{\gamma} \in \operatorname{End} H$, которьй действует на вектор $f \in L^{2}\left(S^{1}, \mathbb{C}^{N}\right)$ поточечньм применением матрицы $\gamma(z) \in \mathrm{GL}(N, \mathbb{C})$ к вектору $f(z) \in \mathbb{C}^{N}$ :

$$
\left(M_{\gamma} f\right)(z):=\gamma(z) f(z) \text {. }
$$

Нетрудно показать, что построенный оператор $M_{\gamma}$ nринадлежит $\mathrm{GL}_{\mathrm{res}}(H)$ для любого $\gamma \in \operatorname{LGL}(N, \mathbb{C})$.

Действительно, пусть $\gamma(z)=\sum_{k \in \mathbb{Z}} \gamma_{k} z^{k}$, где $\gamma_{k} \in \mathrm{L}(N, \mathbb{C})$. Выберем в $H$ базис, задаваемьй функщиями вида $\varepsilon_{i} z^{p}$, где $\left\{\varepsilon_{i}\right\}$ - фиксированньй ортонормированньй базис в $\mathbb{C}^{N}$, а $p \in \mathbb{Z}$. В этом базисе оператор $M_{\gamma}$ имеет матричное представление вида

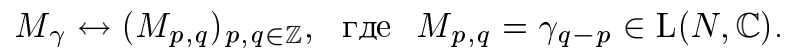

Для того чтобы оператор $M_{\gamma} \in \mathrm{GL}_{\mathrm{res}}(H)$, необходимо и достаточно, чтобы его компоненты, задаваемые отображениями $M_{\gamma}^{+-}: H_{+} \rightarrow H_{-}$и $M_{\gamma}^{-+}: H_{-} \rightarrow H_{+}$, были операторами Гильберта-Шмидта. В терминах матричного представления $\left(M_{p, q}\right)_{p, q \in \mathbb{Z}}$ это означает, что должны вьполняться неравенства

$$
\sum_{p \geqslant 0, q<0}\left\|M_{p, q}\right\|^{2}<\infty \text { и } \sum_{p<0, q \geqslant 0}\left\|M_{p, q}\right\|^{2}<\infty .
$$


Указанные соотношения равносильны неравенству $\sum_{k \in \mathbb{Z}} k\left\|\gamma_{k}\right\|^{2}<\infty$, которое выполняется, если петля $\gamma$ непрерьвно дифференщируема.

Для того чтобы построить вложение в $\mathrm{GL}_{\mathrm{res}}(H)$ группы петель $L G$, где $G$ компактная группа Ли, нужно выбрать в качестве $H$ гильбертово пространство $L^{2}\left(S^{1}, \mathfrak{g}^{\mathbb{C}}\right)$, на котором группа $L G$ действует с помошњю присоединенного представления. Отождествляя алгебру Ли $\mathfrak{g}^{\mathbb{C}}$ с $\mathbb{C}^{N}$ (где $N$ - размерность $\mathfrak{g}$ ) и фиксируя инвариантное скалярное произведение на $\mathfrak{g}$, мы реализуем $L G$ в виде подгрупшы групшы $L \mathrm{U}(N)$. Вложение $\operatorname{LGL}(N, \mathbb{C})$ в $\operatorname{GL}_{\text {res }}(H)$, описанное вьше, переводит $L \mathrm{U}(N)$ в $\mathrm{U}_{\text {res }}(H)$.

Построенное вложение групшы петель $L G$ в $\mathrm{U}_{\mathrm{res}}(H)$ индуцирует изометрическое вложение пространства петель $\Omega G$, наделенного кэлеровой метрикой $g^{1}$ (см. п. 2.1), в грассманово многообразие $\mathrm{Gr}_{\mathrm{res}}(H)$. Наличие указанного вложения позволяет свести исследование гармонических отображений в пространства петель $\Omega G$ к изучению рассмотренных выше гармонических отображений в гильбертов грассманиан $\mathrm{Gr}_{\mathrm{res}}(H)$.

Заметим, что гармонические отображения в пространства петель $\Omega G$ тесно связаны с уравнениями Янга-Миллса на $\mathbb{R}^{4}$. Как показано в работе Атьи [1], пространство модулей $G$-инстантонов на $\mathbb{R}^{4}$ (с фиксированной тривиализацией на бесконечности) можно отождествить с пространством центрированных голоморфных отображений $\mathbb{C P}^{1} \rightarrow \Omega G$ из римановой сферы в $\Omega G$. Указанное отождествление основано на теореме Дональдсона, сводящей описание пространства модулей $G$-инстантонов на $\mathbb{R}^{4} \mathrm{\kappa}$ описанию голоморфных $G$-расслоений на $\mathbb{C P}^{2}$, голоморфно тривиальных на "кресте" $\mathbb{C P}_{\infty}^{1} \cup \mathbb{C P}_{\infty}^{1}$ из “бесконечно удаленных" проективных прямых. Поскольку каждое голоморфное расслоение над $\mathbb{C P}^{1}$ можно задать "гладкой функцией перехода", т.е. отображением из $S^{1} \subset \mathbb{C P}^{1}$ в группу $G$, мы получаем семейство отображений $S^{1} \rightarrow G$, параметризуемых точками $\mathbb{C P}_{\infty}^{1}$, т.е. голоморфное отображение $\mathbb{C P}_{\infty}^{1} \rightarrow \Omega G$. С учетом отождествления Атьи решения полных $G$-уравнений Янга-Миллса на $\mathbb{R}^{4}$ должны отвечать гармоническим отображениям $\mathbb{C P}^{1} \rightarrow \Omega G$.

\section{СПИСОК ЛИТЕРАТУРЫ}

[1] M.F. Atiyah. Instantons in two and four dimensions // Comm. Math. Phys. 1984. V. 93. № 4. P. 437-451.

[2] A. Borel, F. Hirzebruch. Characteristic classes and homogeneous spaces. I // Amer. J. Math. 1958. V. 80. P. 458-538.

[3] F. E. Burstall, J. H. Rawnsley. Twistor Theory for Riemannian Symmetric Spaces With Applications to Harmonic Maps of Riemann Surfaces. Berlin: Springer-Verlag, 1990. (Lecture Notes in Math. V. 1424.)

[4] F. E. Burstall, S. M. Salamon. Tournaments, flags and harmonic maps // Math. Ann. 1987. V. 277. № 2. P. 249-265.

[5] F. E. Burstall, J. C. Wood. The consruction of harmonic maps into complex Grassmannians // J. Differential Geom. 1986. V. 23. № 3. P. 255-297.

[6] $\breve{И}$. Давидов, А. Г. Сергеев. Твисторные пространства и гармонические отображения // УМН. 1993. Т. 48. № 3. С. 3-96.

[7] J.-L. Koszul, B. Malgrange. Sur certaines structures fibrées complexes // Arch. Math. (Basel). 1958. V. 9. P. 102-109.

[8] Э. Прессли, Г. Сигал. Группы петель. М.: Мир, 1990.

[9] А. Г. Сергеев. Кэлерова геометрия пространств петель. М.: МЦНМО, 2001. 
[10] K. Uhlenbeck. Harmonic maps into Lie groups: classical solutions of the chiral model // J. Differential Geom. 1989. V. 30. № 1. P. 1-50.

[11] G. Valli. On the energy spectrum of harmonic 2-spheres in unitary groups // Topology. 1988. V. 27. № 2. P. 129-136.

Математический институт

Поступила в редакцию

им. В.А. Стеклова РАН

03.11 .2004 